\title{
AFLOW-CHULL: Cloud-oriented platform for autonomous phase stability analysis
}

\author{
Corey Oses,${ }^{1}$ Eric Gossett,${ }^{1}$ David Hicks, ${ }^{1}$ Frisco Rose, ${ }^{1}$ Michael J. Mehl,,${ }^{2}$ Eric Perim, ${ }^{1}$ Ichiro Takeuchi,${ }^{3,4}$ \\ Stefano Sanvito, ${ }^{5}$ Matthias Scheffler, ${ }^{6}$ Yoav Lederer, ${ }^{1,7}$ Ohad Levy, ${ }^{1,7}$ Cormac Toher, ${ }^{1}$ and Stefano Curtarolo ${ }^{1,6, *}$ \\ ${ }^{1}$ Department of Mechanical Engineering and Materials Science and Center for Materials Genomics, \\ Duke University, Durham, North Carolina 27708, USA \\ ${ }^{2}$ United States Naval Academy, Annapolis, Maryland 21402, USA \\ ${ }^{3}$ Department of Materials Science and Engineering, \\ University of Maryland, College Park, Maryland 20742-4111, USA \\ ${ }^{4}$ Center for Nanophysics and Advanced Materials, \\ University of Maryland, College Park, Maryland 20742, USA \\ ${ }^{5}$ School of Physics, AMBER and CRANN Institute, Trinity College, Dublin 2, Ireland \\ ${ }^{6}$ Fritz-Haber-Institut der Max-Planck-Gesellschaft, 14195 Berlin-Dahlem, Germany \\ ${ }^{7}$ Department of Physics, NRCN, P.O. Box 9001, Beer-Sheva 84190, Israel
}

(Dated: June 20, 2018)

A priori prediction of phase stability of materials is a challenging practice, requiring knowledge of all energetically-competing structures at formation conditions. Large materials repositories - housing properties of both experimental and hypothetical compounds - offer a path to prediction through the construction of informatics-based, ab-initio phase diagrams. However, limited access to relevant data and software infrastructure has rendered thermodynamic characterizations largely peripheral, despite their continued success in dictating synthesizability. Herein, a new module is presented for autonomous thermodynamic stability analysis implemented within the open-source, ab-initio framework AFLOW. Powered by the AFLUX Search-API, AFLOW-CHULL leverages data of more than 1.8 million compounds currently characterized in the AFLOW.org repository and can be employed locally from any UNIX-like computer. The module integrates a range of functionality: the identification of stable phases and equivalent structures, phase coexistence, measures for robust stability, and determination of decomposition reactions. As a proof-of-concept, thorough thermodynamic characterizations have been performed for more than 1,300 binary and ternary systems, enabling the identification of several candidate phases for synthesis based on their relative stability criterion - including 18 promising $C 15_{b}$-type structures and two half-Heuslers. In addition to a full report included herein, an interactive, online web application has been developed showcasing the results of the analysis, and is located at aflow.org/aflowchull.

\section{INTRODUCTION}

Accelerating the discovery of new functional materials demands an efficient determination of synthesizability. In general, materials synthesis is a multifaceted problem, spanning i. technical challenges, such as experimental apparatus design and growth conditions [1,2], as well as ii. economic and environmental obstacles, including accessibility and handling of necessary components $[3,4]$. Phase stability is a limiting factor. Often, it accounts for the gap between materials prediction and experimental realization. Addressing stability requires an understanding of how phases compete thermodynamically. Despite the wealth of available experimental phase diagrams [5], the number of systems explored represents a negligible fraction of all hypothetical structures [6, 7]. Large materials databases [8-16] enable the construction of calculated phase diagrams, where aggregate structural and energetic materials data is employed. The analysis delivers many fundamental thermodynamic descriptors, including stable/unstable classification, phase coexistence, measures of robust stability, and complete determination of decomposition reactions $[4,17-20]$.

As with all informatics-based approaches, ab-initio phase diagrams require an abundance of data - well-

\footnotetext{
*stefano@duke.edu
}

converged enthalpies from a variety of different phases. Many thermodynamic descriptors computed from the AFLOW.org repository have already demonstrated predictive power in characterizing phase stability [4, 17, 21-34], including one investigation that resulted in the synthesis of two new magnets - the first ever discovered by computational approaches [4]. As exploration embraces more complex systems, such analyses are expected to become increasingly more critical in confining the search space. In fact, prospects for stable ordered phases diminishes with every new component (dimension) despite the growing number of combinations due to i. increased competition with phases of lower dimensionality, e.g., ternary phases additionally compete with stable binary phases [34], and ii. increased competition with disordered (higher entropy) phases [35-37].

To address the challenge, a new module has been implemented in the autonomous, open-source [38] AFLOW (Automatic Flow) framework for ab-initio calculations [21, 23-25, 29, 39-44]. AFLOW-CHULL (AFLOW convex hull) offers a thorough thermodynamic characterization that can be employed locally from any UNIXlike machine, including those running Linux and macOS. Built-in data curation and validation schemes ensure results are sound and properly converged: adhering to proper hull statistics, performing outlier detection, and determining structural equivalence. AFLOW-CHULL is powered by the AFLUX Search-API (application programming interface), which enables access to more 

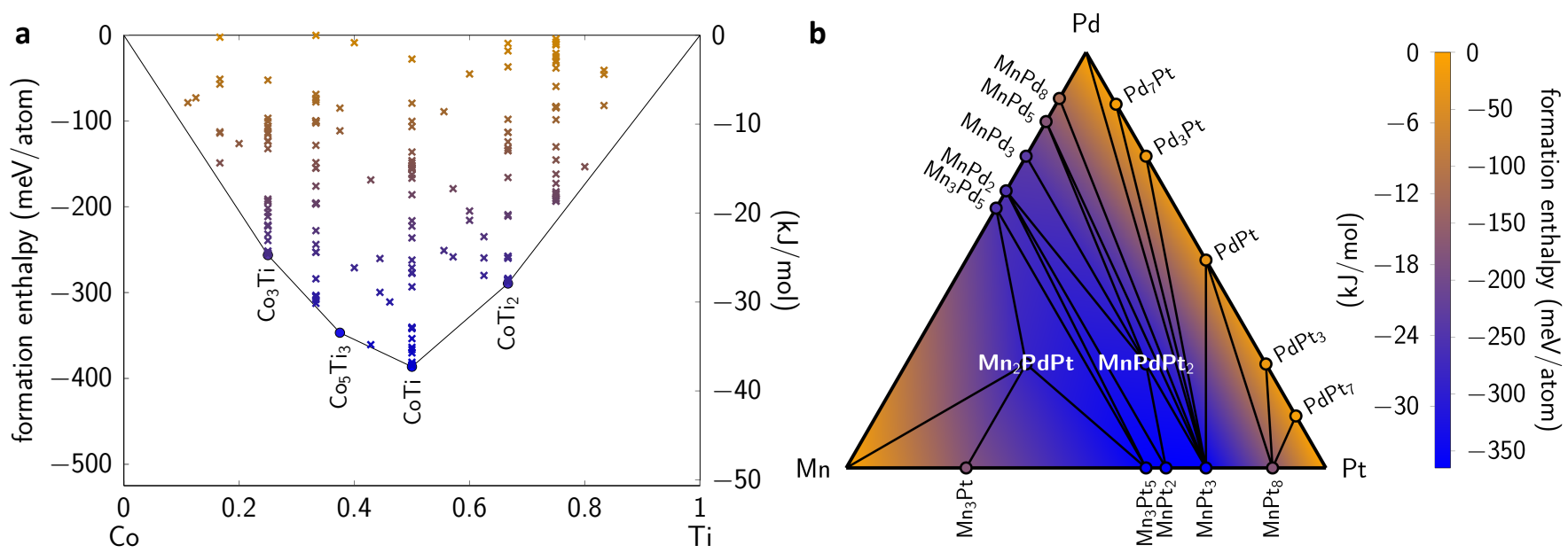

FIG. 1. Example hull illustrations in 2-/3-dimensions as generated by AFLOW-CHULL: (a) Co-Ti and (b) MnPd-Pt.

than 1.8 million compounds from the AFLOW.org repository [11]. With AFLUX integration, data-bindings are flexible enough to serve any materials database, including large heterogeneous repositories such as NoMaD [12].

Several analysis output types have been created to integrate into a variety of design workflows, including plain text and JSON (JavaScript Object Notation) file types. A small set of example scripts have been included demonstrating how to employ AFLOW-CHULL from within a Python environment, much in the spirit of AFLOWSYM [45]. The JSON output also powers an interactive, online web application offering enhanced presentation of thermodynamic descriptors and visualization of 2-/3-dimensional hulls. The application can be accessed through the AFLOW.org portal located at aflow.org/aflowchull.

As a test-bed, the module is applied to all 1.8 million compounds available in the AFLOW.org repository. After enforcing stringent hull convergence criteria, the module resolves a thorough thermodynamic characterization for more than 1,300 binary and ternary systems. Stable phases are screened for previously explored systems and ranked by their relative stability criterion, a dimensionless quantity capturing the effect of the phase on the minimum energy surface [4]. Several promising candidates are identified, including $18 C 15_{b}$-type structures $(F \overline{4} 3 m \# 216)$ and two half-Heuslers. Hence, screening criteria based on these thermodynamic descriptors can accelerate the discovery of new stable phases. More broadly, the design of more challenging materials, including ceramics [46] and metallic glasses [31], benefit from autonomous, integrated platforms like AFLOW-CHULL.

\section{METHODS}

Defining thermodynamic stability. For a multicomponent system at a fixed temperature $(T)$ and pressure $(p)$, the minimum Gibbs free energy $G$ (per atom) defines the thermodynamic equilibrium:

$$
G\left(T, p,\left\{x_{i}\right\}\right)=H-T S
$$

where $x_{i}$ is the atomic concentration of the $i$-species, $H$ is the enthalpy, and $S$ is the entropy. A binary phase $A_{x_{A}} B_{x_{B}}$ is stable at equilibrium with respect to its components $A$ and $B$ if the corresponding formation reaction releases energy:

$$
x_{A} A+x_{B} B \stackrel{\Delta G<0}{\longrightarrow} A_{x_{A}} B_{x_{B}}
$$

where $\Delta G$ is the energy difference between the mixed phase and the sum of its components. Conversely, a positive $\Delta G$ suggests the decomposition of $A_{x_{A}} B_{x_{B}}$ is preferred, and is thus unstable. In general, the magnitude of $\Delta G$ quantifies the propensity for the reaction, and the sign determines the direction.

Relative stability can be visualized on a free-energyconcentration diagram $-\Delta G$ versus $\left\{x_{i}\right\}-$ where $\Delta G$ is depicted as the energetic vertical-distance between $A_{x_{A}} B_{x_{B}}$ and the tie-line connecting $A$ and $B$ endmembers (elemental phases). End-members constitute only a single pathway to formation/decomposition, and all feasible reactions should be considered for systemwide stability. The solution is mathematically equivalent to the construction of the convex hull - the set of the most extreme or "outside" points (Figure 1(a)).

In the zero temperature limit (as is the case for groundstate density functional theory), the entropic term of Equation 1 vanishes, leaving only the formation enthalpy term (per atom) as the driving force:

$$
H_{\mathrm{f}}=H_{A_{x_{A}} B_{x_{B}}}-\left(x_{A} H_{A}+x_{B} H_{B}\right) .
$$

By construction, formation enthalpies of stable elemental phases are zero, which reduces the construction of the convex hull to that of the lower hemisphere. By offsetting 


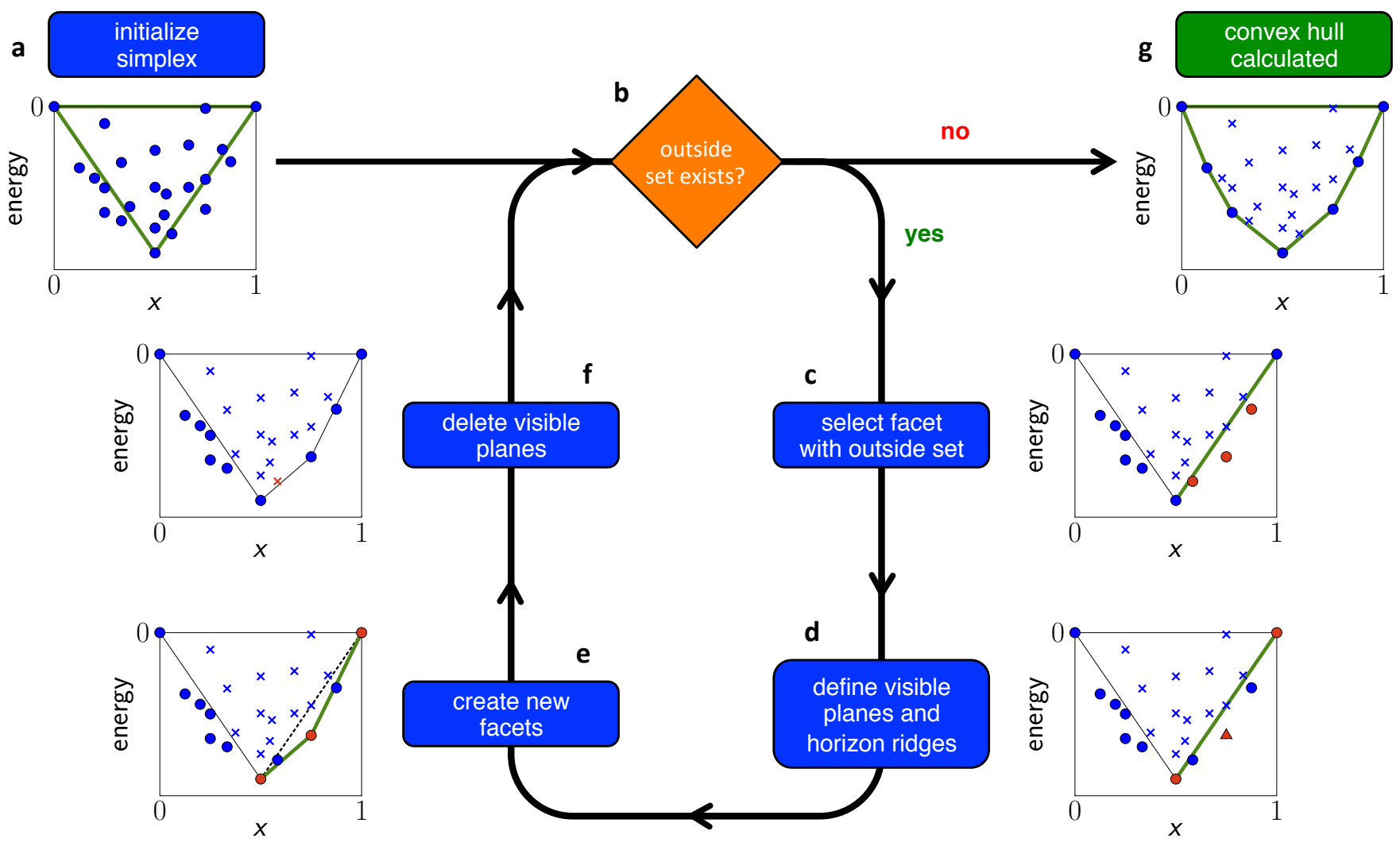

FIG. 2. Illustration of the convex hull construction for a binary system with AFLOW-CHULL. The approach is inspired by the Qhull algorithm [47]. The points on the plot represent structures from the AFLOW.org database [8-11]. (a) and (g) denote the beginning and the end of the algorithm, respectively. (c-f) denote the iterative loop that continues until the condition denoted by (b) is no longer satisfied. Points are marked with crosses if, by that step in the algorithm, they have been determined to be inside the hull, and otherwise are marked with circles. The furthest point from the facet in (d) is distinctly marked with a triangle. Points and facets of interest are highlighted in red and green, respectively.

the enthalpy with that of the elemental phases, $H_{\mathrm{f}}$ quantifies the energy gain from forming new bonds between unlike components ${ }^{1}$, e.g., $A-B$.

The tie-lines connecting stable phases in Figure 1(a) define regions of phase separation where the two phases coexist at equilibrium. The chemical potentials are equal for each component among coexisting phases, implying the common tangent tie-line construction [48, 49]. Phases above a tie-line will decompose into a linear combination of the stable phases that define the tie-line (Figure 4(d)). The Gibbs phase rule [50] dictates the shape of tie-lines for $N$-ary systems, which generalizes to $(N-1)$-dimensional triangles (simplexes) and correspond to facets of the convex hull, e.g., lines in two dimensions (Figure 1(a)), triangles in three dimensions (Figure 1(b)), and tetrahedra in four. The set of equilibrium facets define the $N$-dimensional minimum energy surface.

1 The formation enthalpy is not to be confused with the cohesive energy, which quantifies the energy difference between the phase and its fully gaseous (single atoms) counterpart, i.e., the energy in all bonds.
Hull construction. AFLOW-CHULL calculates the $N$ dimensional convex hull corresponding to an $N$-ary system with an algorithm partially inspired by Qhull [47]. The algorithm is efficient in identifying the most important points for construction of facets, which are treated as hyperplanes instead of boundary-defining inequalities. AFLOW-CHULL uniquely accommodates thermodynamic hulls, i.e., data strictly occupying the lower half hemisphere and defined by stoichiometric coordinates $\left(0 \leq x_{i} \leq 1\right)$. Points corresponding to individual phases are characterized by their stoichiometric and energetic coordinates:

$$
\mathbf{p}=\left[x_{1}, x_{2}, \ldots, x_{N-1}, H_{\mathrm{f}}\right]=\left[\mathbf{x}, H_{\mathrm{f}}\right],
$$

where $x_{N}$ is implicit $\left(\sum_{i} x_{i}=1\right)$. Data preparation includes the $\mathbf{i}$. elimination of phases unstable with respect to end-members (points above the zero $H_{\mathrm{f}}$ tie-line) and ii. organization of phases by stoichiometry and sorted by energy. Through this stoichiometry group structure, all but the minimum energy phases are eliminated for the convex hull calculation.

The workflow is illustrated in Figure 2. AFLOWCHULL operates by partitioning space, iteratively defin- 


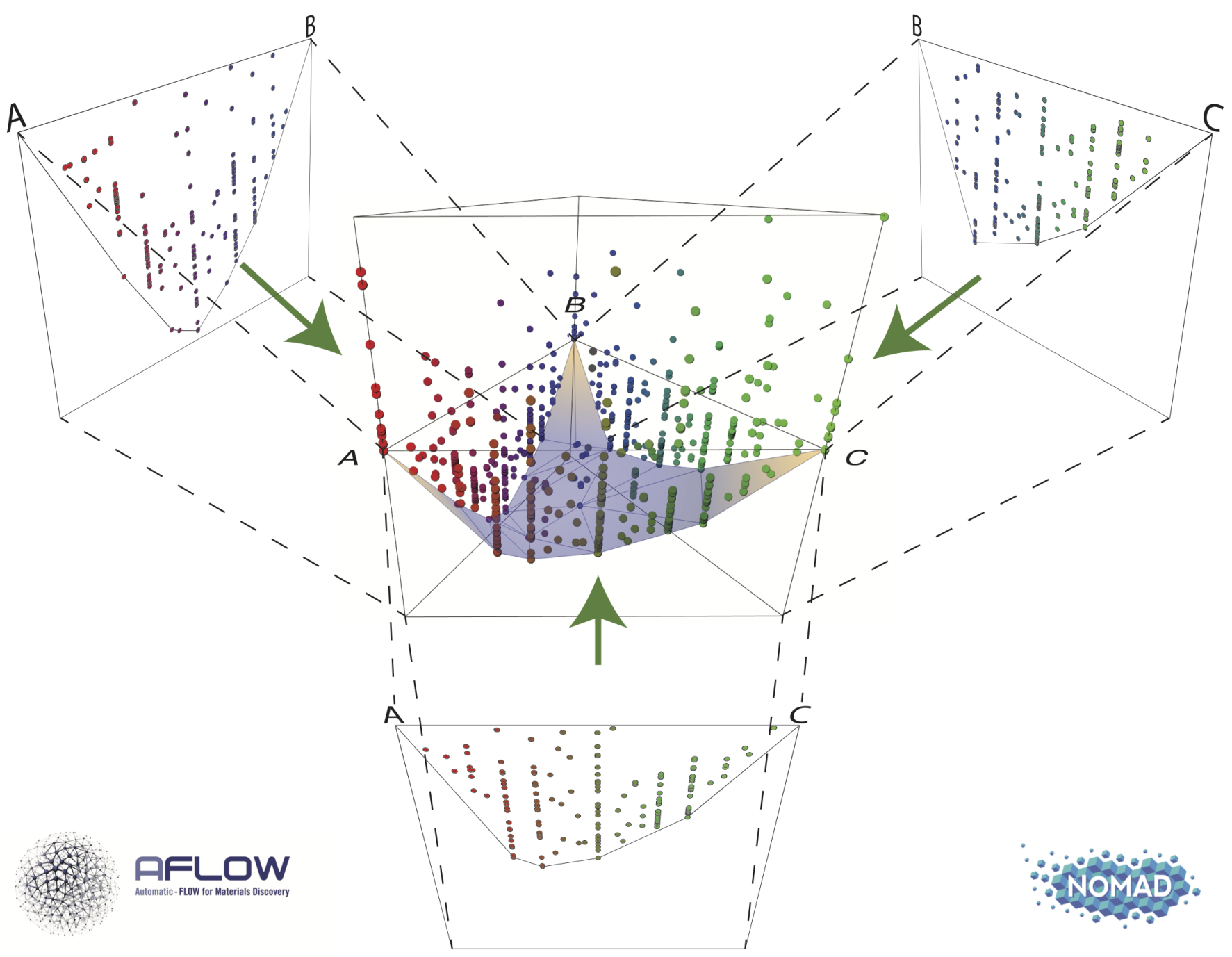

FIG. 3. Illustration of the AFLOW-CHULL iterative hull scheme. The convex hull and associated properties are first calculated for the binary hulls, and then propagated to the ternary hull. This is generalized for $N$-dimensions.

ing "inside" versus "outside" half-spaces until all points are either on the hull or inside of it. First, a simplex is initialized (Figure 2(a)) with the most extreme points: stable end-members and the globally stable mixed phase (lowest energy). A facet is described as:

$$
\mathbf{n} \cdot \mathbf{r}+D=0
$$

where $\mathbf{n}$ is the characteristic normal vector, $\mathbf{r}$ is the position vector, and $D$ is the offset. A general hyperplane is uniquely defined by $N$ points and $k=(N-1)$ corresponding edges $\mathbf{v}_{k}=\mathbf{p}_{k}-\mathbf{p}_{\text {origin }}$. To construct $\mathbf{n}$, AFLOW-CHULL employs a generalized cross product approach [51], where $n_{i \in\{1, \ldots, N\}}$ (unnormalized) is the $i$ row cofactor $\left(C_{i, j=0}\right)$ of the matrix $\mathbf{V}$ containing $\mathbf{v}_{k}$ in its columns:

$$
n_{i}=(-1)^{i+1} M_{i, j=0}\left(\left[\begin{array}{ccc}
\mid & & \mid \\
\mathbf{v}_{1} & \ldots & \mathbf{v}_{k} \\
\mid & & \mid
\end{array}\right]\right)
$$

Here, $M_{i, j=0}(\mathbf{V})$ denotes the $i$-row minor of $\mathbf{V}$, i.e., the determinant of the submatrix formed by removing the $i$-row.

The algorithm then enters a loop over the facets of the convex hull until no points are declared "outside", defined in the hyperplane description by the signed pointplane distance (Figure 2(b)). Each point outside of the hull is singularly assigned to the outside set of a facet (red in Figure 2(c)). The furthest point from each facet - by standard point-plane distance - is selected from the outside set (marked with a triangle in Figure 2(d)). Each neighboring facet is visited to determine whether the furthest point is also outside of it, defining the set of visible planes (green) and its boundary, the horizon ridges (red) (Figure 2(d)). The furthest point is combined with each ridge of the horizon to form new facets (Figure 2(e)). The visible planes - the dotted line in Figure 2(e) - are then removed from the convex hull (Figure 2(f)). The fully constructed convex hull — with 
all points on the hull or inside of it - is summarized in Figure 2(g).

A challenge arises with lower dimensional data in higher dimensional convex hull constructions. For example, binary phases composed of the same species all exist on the same (vertical) plane in three dimensions. A half-space partitioning scheme can make no "inside" versus "outside" differentiation between such points. These ambiguously defined facets ${ }^{2}$ constitute a hull outside the scope of the Qhull algorithm [47]. In the case of three dimensions, the creation of ill-defined facets with collinear edges can result. Hyper-collinearity - planes defined with collinear edges, tetrahedra defined with coplanar faces, etc. - can generally be prescribed by the content (hyper-volume) of the facet. The quantity resolves the length of the line (1-simplex), the area of a triangle (2-simplex), the volume of a tetrahedron (3-simplex), etc., and is calculated for a simplex of $N$-dimensions via the Cayley-Menger determinant [52]. Both vertical and content-less facets are problematic for thermodynamic characterizations, particularly when calculating hull distances, which require facets within finite energetic distances and well-defined normals.

A dimensionally-iterative scheme is implemented in AFLOW-CHULL to solve the issue. It consecutively calculates the convex hull for each dimension (Figure 3). In the case of a ternary hull, the three binary hulls are calculated first, and the relevant thermodynamic data is extracted and then propagated forward. Though vertical and content-less facets are still created in higher dimensions, no thermodynamic descriptors are extracted from them. To optimize the calculation, only stable binary structures are propagated forward to the ternary hull calculation, and this approach is generalized for $\mathrm{N}$ dimensions. The scheme is automatically chosen for thermodynamic hulls, resorting back to the general convex hull algorithm otherwise.

Thermodynamic data. Structural and energetic data employed to construct the convex hull is retrieved from the AFLOW.org [8-11] repository, which currently contains approximately 1.8 million compounds and 180 mil- lion calculated properties. The database is generated by the autonomous, ab-initio framework AFLOW [21, 2325, 29, 39-44] following the AFLOW Standard for highthroughput materials science calculations [10]. In particular, calculations are performed with VASP (Vienna $\underline{A} b$ initio Simulation Package) [54-57]. Wavefunctions are accurately represented by a large basis set, including all terms with kinetic energy up to a threshold larger by a factor of 1.4 than the recommended defaults. AFLOW also leverages a large $\mathbf{k}$-point mesh - as standardized by a k-points-per-reciprocal-atom scheme [10] — which is critical for convergence and reliability of calculated properties. Investigations show that the AFLOW Standard of at least 6,000 $\mathbf{k}$-points-per-reciprocal-atom for structural relaxations and 10,000 for the static calculations ensures robust convergence of the energies to within one $\mathrm{meV}$ /atom in more than $95 \%$ of systems (including metals which suffer from the discontinuity in the occupancy function at zero temperature), and within three meV/atom otherwise [58].

Special consideration is taken for the calculation of $H_{\mathrm{f}}$. The reference energies for the elemental phases are calculated and stored in the LIB1 catalog for unary phases in the AFLOW.org repository, and include variations for different functionals and pseudopotentials. For consistency, AFLOW-CHULL only employs data calculated with the Perdew-Burke-Ernzerhof Generalized Gradient Approximation functional and pseudopotentials calculated with the projector augmented wave method (PAWPBE) $[59,60]$. It is possible to encounter stable (lowest energy) elemental phases with energies that differ from the reference (LIB1) by a fraction of $\mathrm{meV} /$ atom, which is the result of duplicate entries (by relaxation or otherwise) as well as reruns with new parameters, e.g., a denser kpoint mesh. To avoid any issues with the convex hull calculation, the algorithm fixes the half-space plane at zero. However, a "warning" is prompted in the event that the stable elemental phase differs from the reference energy by more than $15 \mathrm{meV}$ /atom.

Data is retrieved via the AFLUX Search-API [11], designed for accessing property-specific datasets efficiently. The following is an example of a relevant request:

$$
\text { http://aflowlib.duke.edu/search/API/?species(Mn,Pd), nspecies(2), }{ }^{*}, \operatorname{paging}(0)
$$

where http://aflowlib.duke.edu/search/API/ is the URL for the AFLUX server and $\operatorname{species}(\mathrm{Mn}, \mathrm{Pd})$, nspecies(2), ${ }^{*}$, paging(0) is the query. Specifically, species(Mn, Pd) queries for any entry containing the elements $\mathrm{Mn}$ or $\mathrm{Pd}$, nspecies(2) limits the search to binaries only, ${ }^{*}$ returns the data for all available

\footnotetext{
2 The issue is generally prescribed when a set of $d+1$ points (or more) define a $(d-1)$-flat [47].
}

fields, and paging(0) amalgamates all data into a single response without paginating (warning, this can be a large quantity of data). Such queries are constructed combinatorially for each dimension, e.g., a general ternary hull $A B C$ constructs the following seven queries: species $(A)$, species $(B)$, and species $(C)$ with nspecies(1), species $(A, B)$, species $(A, C)$, and $\operatorname{species}(B, C)$ with nspecies(2), and species $(A, B, C)$ with nspecies(3).

Validation schemes. Various statistical analyses and data curation procedures are employed automatically 

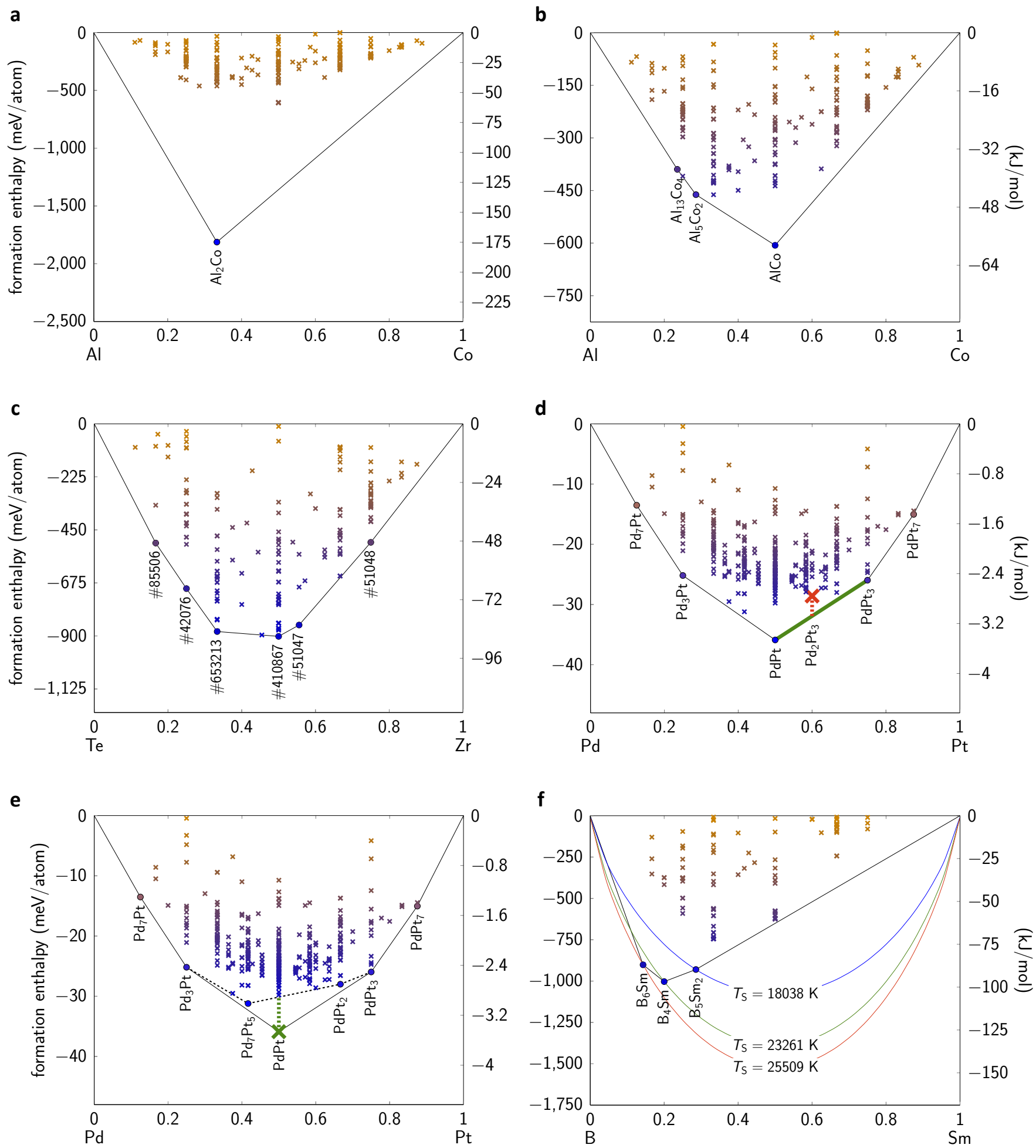

FIG. 4. Illustration of various automated convex hull analyses in AFLOW-CHULL. (a) A plot showing an egregious outlier in the Al-Co convex hull. (b) The corrected Al-Co convex hull (with the outlier removed). (c) The Te-Zr convex hull with the traditional compound labels replaced with the corresponding ICSD number designations as determined by a structure comparison analysis. If multiple ICSD entries are found for the same stoichiometry, the lowest ICSD entry is chosen (chronologically reported, usually). (d) The decomposition energy of $\mathrm{Pd}_{2} \mathrm{Pt}_{3}$ is plotted in red, and highlighted in green is the equilibrium facet directly below it. The facet is defined by ground state phases $\mathrm{PdPt}_{3}$ and PdPt. (e) The stability criterion $\delta_{\mathrm{sc}}$ is plotted in green, with the pseudo-hull plotted with dashed lines. (f) The B-Sm convex hull plotted with the ideal "iso-max-latent-heat" lines of the grand-canonical ensemble $[29,53]$ for the ground state structures. 
by AFLOW-CHULL to maximize fidelity. At a minimum, each binary hull must contain 200 structures to ensure a sufficient sampling size for inference. There is never any guarantee that all stable structures have been identified [29, 61], and convergence is approached with larger datasets. With continued growth of LIB3 (ternary phases) and beyond, higher dimensional parameters will be incorporated, though it is expected that the parameters are best defined along tie-lines (versus tie-surfaces). A comprehensive list of available alloys and structure counts are included in the Supporting Information.

Outlier detection. In addition to having been calculated with a standard set of parameters [10], database entries incorporated in the convex hull calculation should also be similarly well-converged. Prior to the injection of new entries into the AFLOW.org database, various verification tests are employed to ensure convergence, including an analysis of the relaxed structure's stress tensor [11]. Issues stemming from poor convergence and failures in the functional parameterization $[17,61]$ can dramatically change the topology of the convex hull, resulting in contradictions with experiments. Hence, an outlier detection algorithm is applied before the hull is constructed: structures are classified as outliers and discarded if they have energies that fall well below the first quartile by a multiple of the interquartile range (conservatively set to 3.25 by default) [62]. Only points existing in the lower half-space (phases stable against endmembers) are considered for the outlier analysis, and hence systems need to show some miscibility, i.e., at least four points for a proper interquartile range determination. Despite its simplicity, the interquartile range is the preferred estimate of scale over other measures such as the standard deviation or the median absolute deviation which require knowledge of the underlying distribution (normal or otherwise) [63]. An example hull (Al-Co) showing an outlier is plotted in Figure 4(a) and the corrected hull with the outlier removed is presented in Figure 4(b).

Duplicate detection. A procedure for identifying duplicate entries is also employed. By database construction, near-exact duplicates of elemental phases exist in LIB2, which is created spanning the full range of compositions for each alloy system (including elemental phases). These degenerate entries are easily detected and removed automatically by comparing composition, prototype, and formation enthalpy. Other structures may have been created distinctly, but converge to duplicates via structural relaxation. These equivalent structures are detected via AFLOW-XTAL-MATCH (AFLOW crystal match) [64], which determines structural/material uniqueness via the Burzlaff criteria [65]. To compare two crystals, a commensurate representation between structures is resolved by i. identifying common unit cells, ii. exploring cell orientations and origin choices, and iii. matching atomic positions. For each description, the structural similarity is measured by a composite misfit quantity based on the lattice deviations and mismatch of the mapped atomic positions, with a match occurring for sufficiently small misfit values $(<0.1)$. Depending on the size of the structures, the procedure can be quite expensive. As such, it is applied (automatically) to find only duplicate stable structures. Candidates are first screened by composition, space group, and formation enthalpies (must be within $15 \mathrm{meV} /$ atom of the relevant stable configuration). The identification of duplicate stable phases has proven quite fruitful, enabling rapid identification of potentially unexplored stable structures, particularly when comparing with the AFLOW.org ICSD (Inorganic $\underline{\text { Crystal }}$ Structure Database) catalog [66, 67]. The analysis is depicted in Figure 4(c), where the Te-Zr convex hull is plotted with the compound labels replaced with the corresponding ICSD number designation.

Thermodynamic descriptors. A wealth of properties can be extracted from the convex hull construction beyond a simple determination of stable/unstable phases. For unstable structures, the energy driving the decomposition reaction $\Delta H_{\mathrm{f}}$, i.e., the energetic vertical-distance to the hull depicted in Figure 4(d), serves as a useful metric for quasi-stability. Without the temperature and pressure contributions to the energy, near-stable structures should also be considered (meta-)stable candidates, e.g., those within $k_{\mathrm{B}} T=25 \mathrm{meV}$ (room temperature) of the hull. Highly disordered systems can be realized with even larger distances [17, 68].

To calculate $\Delta H_{\mathrm{f}}$ of phase $\mathbf{p}$ (Equation 4), AFLOWCHULL first resolves the energy of the hull $H_{\text {hull }}$ at stoichiometric coordinates $\mathbf{x}$, and then offsets it by the phase's formation enthalpy $H_{\mathrm{f}}$ :

$$
\Delta H_{\mathrm{f}}[\mathbf{p}]=H_{\text {hull }}[\mathbf{x}]-H_{\mathrm{f}} .
$$

The procedure is depicted in Figure 4(d), which involves identifying the facet (highlighted in green) that bounds $\mathbf{x}$ and thus defines $H_{\text {hull }}(\mathbf{x})$. Despite limitations of the hyperplane description of facets (Equations 5 and 6), which lacks boundaries in the stoichiometric axes [17], the appropriate facet is identified as that which minimizes the distance to the zero $H_{\mathrm{f}}$ tie-line at $\mathbf{x}$ :

$$
H_{\text {hull }}[\mathbf{x}]=-\min _{\text {facets } \in \text { hull }}\left|n_{N}^{-1}\left(D+\sum_{i=1}^{N-1} n_{i} x_{i}\right)\right| .
$$

Vertical facets and those showing hyper-collinearity (having no content) are excluded from the calculation. By this convention, unstable phases have negative distances to the hull, indicative of a decomposition reaction (compare with Equations 2 and 9).

Furthermore, the $l$ coefficients of the balanced decomposition reaction are derived to yield the full equation. The decomposition of an $N$-ary phase into $l-1$ stable phases defines an $(l \times N)$-dimensional chemical composition matrix $\mathbf{C}$, where $C_{j, i}$ is the signed number of atoms per formula unit of the $i$-species of the $j$-phase (the first of which is the unstable mixed phase). Take, for example, the decomposition reaction presented in Figure 4(d):

$$
N_{1} \mathrm{Pd}_{2} \mathrm{Pt}_{3} \rightarrow N_{2} \mathrm{PdPt}+N_{3} \mathrm{PdPt}_{3},
$$


where $N_{j}$ is the balanced chemical coefficient for the $j$ phase. In this case, $\mathbf{C}$ is defined as:

$$
\left[\begin{array}{cc}
N_{\mathrm{Pd}} \in \mathrm{Pd}_{2} \mathrm{Pt}_{3} & N_{\mathrm{Pt}} \in \mathrm{Pd}_{2} \mathrm{Pt}_{3} \\
-N_{\mathrm{Pd}} \in \mathrm{PdPt} & -N_{\mathrm{Pt}} \in \mathrm{PdPt} \\
-N_{\mathrm{Pd}} \in \mathrm{PdPt}_{3} & -N_{\mathrm{Pt}} \in \mathrm{PdPt}_{3}
\end{array}\right]=\left[\begin{array}{cc}
2 & 3 \\
-1 & -1 \\
-1 & -3
\end{array}\right],
$$

where a negative sign differentiates the right hand side of the equation from the left. Ref. 69 shows that $N_{j}$ can be extracted from the null space of $\mathbf{C}$. AFLOW-CHULL accesses the null space via a full $\mathbf{Q R}$ decomposition of C, specifically employing a general Householder algorithm [70]. The last column of the $(l \times l)$-dimensional $\mathbf{Q}$ orthogonal matrix spans the null space $\mathbf{N}$ :

$$
\mathbf{Q}=\left[\begin{array}{ccc}
\mid & \mid & 0.53452 \\
\mathbf{q}_{1} & \mathbf{q}_{2} & 0.80178 \\
\mid & \mid & 0.26726
\end{array}\right] .
$$

By normalizing $\mathbf{N}$ such that the first element $N_{1}=$ 1, the approach yields $N_{2}=1.5$ and $N_{3}=0.5$, which indeed balances Equation 9. These coefficients can be used to verify the energetic distance $\Delta H_{\mathrm{f}}$ observed in Figure 4(d). The formation enthalpies of $\mathrm{Pd}_{2} \mathrm{Pt}_{3}, \mathrm{PdPt}$, and $\mathrm{PdPt}_{3}$ are $-286 \mathrm{meV} /(10$ atoms $)$, $-72 \mathrm{meV} /(2$ atoms $)$, and $-104 \mathrm{meV} /(4$ atoms $)$, respectively. Here, $\Delta H_{\mathrm{f}}$ is calculated as:

$$
\begin{aligned}
1.5 H_{\mathrm{f}}[\mathrm{PdPt}]+0.5 H_{\mathrm{f}}\left[\mathrm{PdPt}_{3}\right] & -H_{\mathrm{f}}\left[\mathrm{Pd}_{2} \mathrm{Pt}_{3}\right] \\
& =-3 \mathrm{meV} / \text { atom. }
\end{aligned}
$$

For a given stable structure, AFLOW-CHULL automatically determines the phases with which it is in equilibrium. For instance, $\mathrm{PdPt}$ is in two-phase equilibria with $\mathrm{Pd}_{3} \mathrm{Pt}$ as well as with $\mathrm{PdPt}_{3}$ (Figure 4(d)). Phase coexistence plays a key role in defining a descriptor for precipitate-hardened superalloys. Candidates are chosen if a relevant composition is in two-phase equilibrium with the host matrix, suggesting that the formation of coherent precipitates in the matrix is feasible [17, 71].

An analysis similar to that quantifying instability $\left(\Delta H_{\mathrm{f}}\right)$ can be performed to determine the robustness of stable structures. The stability criterion $\delta_{\mathrm{sc}}$ is defined as the distance of a stable structure from the pseudo-hull constructed without it (Figure 4(e)). Its calculation is identical to that of the $\Delta H_{\mathrm{f}}$ for the pseudo-hull (Equations 7 and 8). This descriptor quantifies the effect of the structure on the minimum energy surface, as well as the structure's susceptibility to destabilization by a new phase that has yet to be explored. As with the decomposition analysis, $\delta_{\mathrm{sc}}$ also serves to anticipate the effects of temperature and pressure on the minimum energy surface. The descriptor played a pivotal role in screening Heusler structures for new magnetic systems [4]. $\delta_{\mathrm{sc}}$ calls for the recalculation of facets local to the structure and all relevant duplicates as well, thus employing the results of the structure comparison protocol for stable structures.

Furthermore, AFLOW-CHULL can plot the entropic temperature envelopes characterizing nucleation in hyper-thermal synthesis methods for binary systems [53]. The entropic temperature is the ratio of the formation enthalpy to the mixing entropy for an ideal solution a simple quantification for the resilience against disorder [29]. The ideal "iso-max-latent-heat" lines shown in Figure 4(f) try to reproduce the phase's capability to absorb latent heat, which can promote its nucleation over more stable phases when starting from large Q reservoirs/feedstock. The descriptor successfully predicts the synthesis of $\mathrm{SmB}_{6}$ over $\mathrm{SmB}_{4}$ with hyper-thermal plasma co-sputtering $[29,53]$.

\section{RESULTS}

Analysis output. Following the calculation of the convex hull and relevant thermodynamic descriptors, AFLOW-CHULL automatically generates a PDF file summarizing the results. Included in the PDF are $\mathbf{i}$. an illustration of the convex hull as shown in Figure 1 (for binary and ternary systems) and ii. a report with the aforementioned calculated thermodynamic descriptors an excerpt is shown in Figure 5.

In the illustrations, color is used to differentiate points with different enthalpies and indicate depth of the facets (3-dimensions). The report includes entry-specific data from the AFLOW.org database (prototype, auid, original and relaxed space groups, spin, formation enthalpy $H_{\mathrm{f}}$, and entropic temperature $T_{\mathrm{S}}$ ) as well as calculated thermodynamic data (distance to the hull $\Delta H_{\mathrm{f}}$, the balanced decomposition reaction for unstable phases, the stability criterion $\delta_{\mathrm{sc}}$ for stable phases, and phases in coexistence). Stable phases (and those that are structurally equivalent) are highlighted in green, and similar phases (comparing relaxed space groups) are highlighted in orange. Links are also incorporated in the report, including external hyperlinks to entry pages on AFLOW.org (see prototypes) and internal links to relevant parts of the report (see decomposition reaction and $N$-phase equilibria). Internal links are also included on the convex hull illustration (see Supporting Information). The information is provided in the form of plain text and JSON files. Keys and format are explained in the Appendix.

Web application. A modern web application has been developed to provide an enhanced, command-line-free platform for AFLOW-CHULL. The project includes a rich feature set consisting of binary and ternary convex hull visualizations, AFLOW.org entry data retrieval, and a convex hull comparison interface. The application is divided into four components: the periodic table, the visualization viewport, the selected entries list, and the comparison page.

The periodic table component is displayed. Hulls can be queried by selecting/typing in the elemental combination. As elements are added to the search, the periodic table reacts to the query depending on the reliability of the hull: green (fully reliable, $N_{\text {entries }} \geq 200$ ), orange (potentially reliable, $100 \leq N_{\text {entries }}<200$ ), 


\begin{tabular}{|c|c|c|c|c|c|c|c|}
\hline prototype & auid & $\begin{array}{c}\text { original } \\
\text { space group }\end{array}$ & $\begin{array}{c}\text { relaxed } \\
\text { space group }\end{array}$ & $\begin{array}{c}\text { spin } \\
\left(\mu_{\mathrm{B}} / \text { atom }\right)\end{array}$ & $\begin{array}{c}H_{\mathrm{f}} \\
(\mathrm{meV} / \text { atom })\end{array}$ & $T_{\mathrm{S}}(\mathrm{K})$ & $\begin{array}{c}\Delta H_{\mathrm{f}} \\
(\mathrm{meV} / \mathrm{atom})\end{array}$ \\
\hline
\end{tabular}

$\mathrm{Ag}_{4} \mathrm{AuCd}$

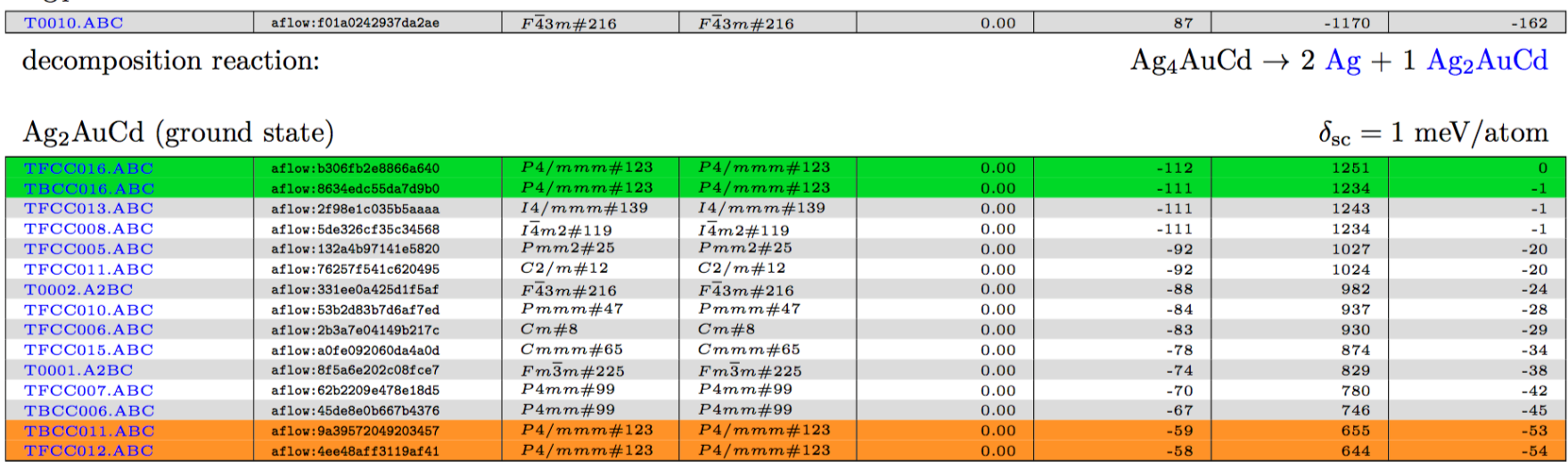

3-phase equilibria:

$\mathrm{Ag}-\mathrm{Ag}_{4} \mathrm{Cd}-\mathrm{Ag}_{2} \mathrm{AuCd}, \mathrm{Ag}-\mathrm{Ag}_{3} \mathrm{Au}-\mathrm{Ag}_{2} \mathrm{AuCd}, \mathrm{Ag}_{4} \mathrm{Cd}-\mathrm{Ag}_{3} \mathrm{Cd}-\mathrm{Ag}_{2} \mathrm{AuCd}$, $\mathrm{Ag}_{3} \mathrm{Au}-\mathrm{AgAu}-\mathrm{Ag}_{2} \mathrm{AuCd}, \mathrm{Ag}_{3} \mathrm{Cd}-\mathrm{Ag}_{2} \mathrm{AuCd}-\mathrm{Au}_{2} \mathrm{Cd}_{3}, \mathrm{AgAu}-\mathrm{Ag}_{2} \mathrm{AuCd}-\mathrm{AgAu} \mathrm{C}_{2} \mathrm{Cd}$, $\mathrm{Ag}_{2} \mathrm{AuCd}-\mathrm{AgAu}_{2} \mathrm{Cd}-\mathrm{AuCd}$, and $\mathrm{Ag}_{2} \mathrm{AuCd}-\mathrm{AuCd}-\mathrm{Au}_{2} \mathrm{Cd}_{3}$

FIG. 5. Excerpt from the Ag-Au-Cd thermodynamic analysis report. The document is generated automatically by AFLOW-CHULL and showcases entry-specific data from the AFLOW.org database as well as calculated thermodynamic descriptors. Structures highlighted in green are structurally equivalent stable structures, and those in orange are structurally similar (same relaxed space group). The working document includes a variety of links, including hyperlinks to the entry page of each phase (see prototypes) and links to relevant parts of the report (see decomposition reaction and $N$-phase equilibria).

red (unreliable, $N_{\text {entries }}<100$ ), and gray (unavailable, $\left.N_{\text {entries }}=0\right)$. Once a selection is made, the application loads the visualization viewport triggering a redirect to the URL endpoint of the selected hull, e.g., /hull/AlHfNi. The URL is ubiquitous and can be individually shared/cited.

When a binary convex hull is selected, the viewport reveals a traditional 2-dimensional plot (Figure 6(a)), while a ternary hull yields a 3-dimensional visualization (Figure $6(\mathrm{~b}))$. The scales of both are tunable, and the 3 dimensional visualization offers mouse-enabled pan and zoom.

Common to both types is the ability to select and highlight points. When a point is selected, its name will appear within the sidebar. The information component is populated with a grid of cards containing properties of each selected point (entry), including a link to the AFLOW.org entry page (Figure 6(d)).

The application environment stores all previously selected hulls, which are retrievable via the hull comparison component (Figure 6(c)). On this page each hull visualization is displayed as a card on a grid. This grid serves as both a history and a means to compare hulls.

Candidates for synthesis. To demonstrate the capability of AFLOW-CHULL, all binary and ternary systems in the AFLOW.org repository are explored for ones yielding well-converged thermodynamic properties. Since reliability constraints are built-in, all potential elemental combinations can be attempted rapidly and effortlessly.
Across all catalogs present in the database, there exist materials composed of 86 elements, including: $\mathrm{H}, \mathrm{He}, \mathrm{Li}$, $\mathrm{Be}, \mathrm{B}, \mathrm{C}, \mathrm{N}, \mathrm{O}, \mathrm{F}, \mathrm{Ne}, \mathrm{Na}, \mathrm{Mg}, \mathrm{Al}, \mathrm{Si}, \mathrm{P}, \mathrm{S}, \mathrm{Cl}, \mathrm{Ar}$, $\mathrm{K}, \mathrm{Ca}, \mathrm{Sc}, \mathrm{Ti}, \mathrm{V}, \mathrm{Cr}, \mathrm{Mn}, \mathrm{Fe}, \mathrm{Co}, \mathrm{Ni}, \mathrm{Cu}, \mathrm{Zn}, \mathrm{Ga}, \mathrm{Ge}$, As, Se, Br, Kr, Rb, Sr, Y, Zr, Nb, Mo, Tc, Ru, Rh, Pd, Ag, Cd, In, Sn, Sb, Te, I, Xe, Cs, Ba, La, Ce, Pr, Nd, Pm, Sm, Eu, Gd, Tb, Dy, Ho, Er, Tm, Yb, Lu, Hf, Ta, W, Re, Os, Ir, Pt, Au, Hg, Tl, Pb, Bi, Ac, Th, and Pa. Hulls are automatically eliminated if systems i. are unreliable based on count (fewer than 200 entries among binary combinations), and ii. show significant immiscibility (fewer than 50 points below the zero $H_{\mathrm{f}}$ tie-line). The analysis resulted in the full thermodynamic characterization of 493 binary and 861 ternary systems. The complete set of results are provided in the Supporting Information.

Leveraging the JSON outputs, reliable hulls are further explored for new stable phases. Phases are first screened (eliminated) if an equivalent structure exists in the AFLOW.org ICSD catalog, and candidates are sorted by their relative stability criterion, i.e., $\delta_{\mathrm{sc}} / H_{\mathrm{f}}$. This dimensionless quantity captures the effect of the phase on the minimum energy surface relative to its depth, enabling comparisons across hulls.

The top 25 most stable binary and ternary phases are presented in Tables 1 and 2, respectively, for which extended analysis is performed based on information stored

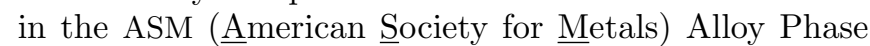
Diagram database [5]. The ASM database is the largest 
a

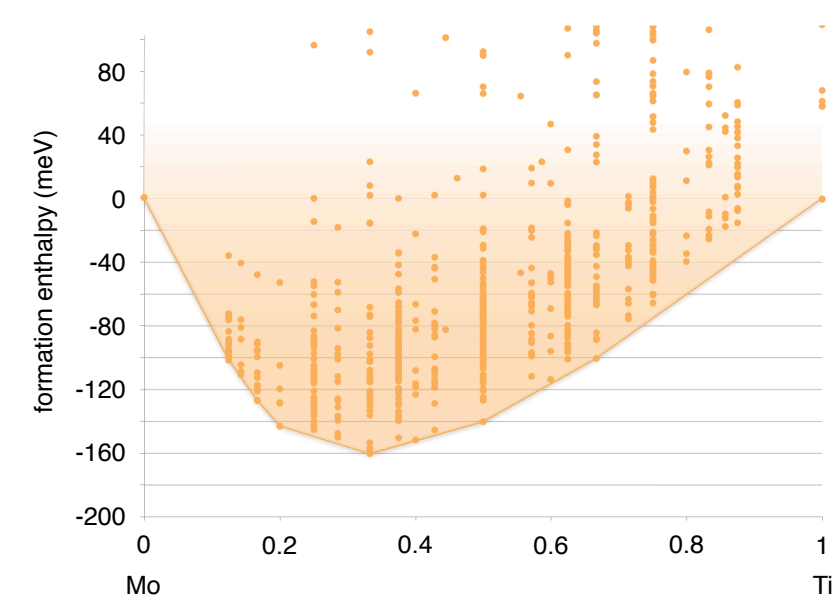

b

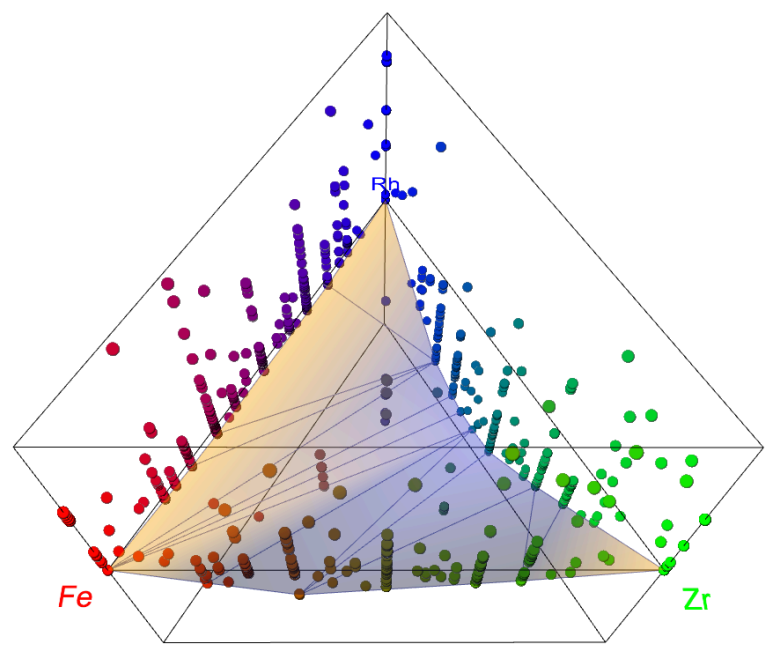

C

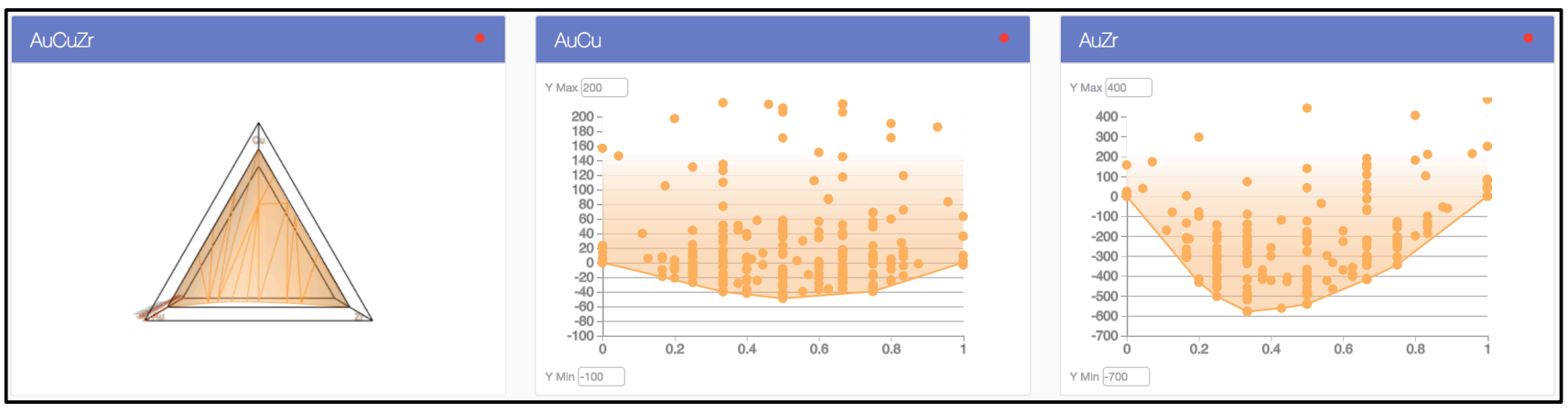

d

\begin{tabular}{|c|c|c|c|c|c|c|c|}
\hline \multirow{2}{*}{\multicolumn{2}{|c|}{$\begin{array}{c}\text { Add a Hull... } \\
\text { Selected Points }\end{array}$}} & \multirow{2}{*}{\multicolumn{2}{|c|}{ aflow:35cf7f959780e51f }} & & \multirow{2}{*}{\multicolumn{2}{|c|}{ aflow:68981f8fe574a335 }} & \\
\hline & & & & & & & \\
\hline Au1 & - & Au1 & LIB2 & cubic & Au2 & LIB2 & tetragonal \\
\hline \multirow{5}{*}{ Au2 } & $\bullet$ & Compound & Catalog & Lattice & Compound & Catalog & Lattice \\
\hline & & 229 & 18.054 & 23.088 & 141 & 16.510 & 156.456 \\
\hline & & Space group & Density $\left(\mathrm{g} / \mathrm{cm}^{3}\right)$ & Enthalpy (meV) & Space group & Density $\left(\mathrm{g} / \mathrm{cm}^{3}\right)$ & Enthalpy (meV) \\
\hline & & MORE INFO & & & MORE INFO & & \\
\hline & & REMOVE & & & REMOVE & & \\
\hline
\end{tabular}

FIG. 6. The convex hull web application powered by AFLOW-CHULL. (a) An example 2-dimensional convex hull illustration (Mo-Ti). (b) An example 3-dimensional convex hull illustration (Fe-Rh-Zr). (c) The comparison component of the hull application. Each hull visualization is displayed as part of a grid of cards. From this page, new hulls can be added to the store by typing a query within the search box in the sidebar. (d) The information component of the hull application. Pertinent thermodynamic data for selected points is displayed within the grid of cards. Each card includes a link to the AFLOW.org entry page and includes the option to remove a point. As points are selected within the visualization, more cards will be added to the grid.

of its kind, aggregating a wealth of experimental phase diagram information: 40,300 binary and ternary alloy phase diagrams from over 9,000 systems. Upon searching the ASM website, many binary systems from Table 1 are unavailable and denoted by the symbol ${ }^{\dagger}$. Among those that are available, some stable phases have already been observed, including $\mathrm{OsY}_{3}$ and $\mathrm{Be}_{5} \mathrm{Pt}$. For $\mathrm{AgPt}$, $\mathrm{MnRh}, \mathrm{AgAu}$, and PtRh the composition is successfully predicted, but polymorphs (structurally distinct phases) are observed instead. For all other phases on the list, the composition has not been observed. The discrepancy may be isolated to the phase, or indicative of a more ex- 


\begin{tabular}{|c|c|c|c|c|}
\hline compound & auid & relaxed space group & $\delta_{\mathrm{sc}} / H_{\mathrm{f}}$ & comparison with ASM Alloy Phase Diagrams [5] \\
\hline $\mathrm{Hf}_{5} \mathrm{~Pb}^{\dagger}$ & aflow:38ecc639e4504b9d & $P 4 / m m m \# 123$ & $78 \%$ & no diagram \\
\hline $\mathrm{AgIn}_{3}$ & aflow: $11 \mathrm{ba} 11 \mathrm{a} 3 \mathrm{ee} 157 \mathrm{f} 2 \mathrm{e}$ & $\mathrm{P}_{3} / \mathrm{mmc} \# 194$ & $54 \%$ & $\begin{array}{l}\text { composition not found, nearest are } \mathrm{AgIn}_{2} \text { (space group } \\
I 4 / \mathrm{mcm}, \Delta H_{\mathrm{f}}=-53 \mathrm{meV} / \text { atom) and In (space group } \\
I 4 / \mathrm{mmm} \text { ) }\end{array}$ \\
\hline $\mathrm{Hf}_{3} \mathrm{In}_{4}^{\dagger}$ & aflow : 1 da 75 eb5f 31 b6dd5 & $P 4 / m b m ~ \# 127$ & $45 \%$ & no diagram \\
\hline $\mathrm{AsTc}_{2}^{\dagger}$ & flow: 66 dda 41 a34fe3ad 6 & $C 2 / m \# 12$ & $41 \%$ & no diagram \\
\hline $\mathrm{MoPd}_{8}$ & aflow: $57 \mathrm{e} 1 \mathrm{a} 1246 \mathrm{f} 813 \mathrm{f} 27$ & $I 4 / \mathrm{mmm} \# 139$ & $40 \%$ & $\begin{array}{l}\text { composition not found, nearest are } \mathrm{Mo}_{0.257} \mathrm{Pd}_{0.743} \text { (space } \\
\text { group } F m \overline{3} m \text {, POCC structure) and } \mathrm{Pd} \text { (space group } \\
F m \overline{3} m)\end{array}$ \\
\hline $\mathrm{Ga}_{4} \mathrm{Tc}^{\dagger}$ & aflow: $32051219452 \mathrm{f} 8 \mathrm{e} 0 \mathrm{f}$ & $\operatorname{Im} \overline{3} m \# 229$ & $39 \%$ & no diagram \\
\hline $\mathrm{Pd}_{8} \mathrm{~V}$ & aflow : $7 \mathrm{bd} 140 \mathrm{~d} 7 \mathrm{~b} 4 \mathrm{c} 65 \mathrm{bc} 1$ & $I 4 / \mathrm{mmm} \# 139$ & $36 \%$ & $\begin{array}{l}\text { composition not found, nearest are } \mathrm{V}_{0.1} \mathrm{Pd}_{0.9} \text { (space } \\
\text { group } F m \overline{3} m, \text { POCC structure) and } \mathrm{VPd}_{3} \text { (space group } \\
I 4 / m m m, \Delta H_{\mathrm{f}}=-6 \mathrm{meV} / \text { atom) }\end{array}$ \\
\hline $\mathrm{InSr}_{3}$ & aflow: e7ed70c4711eb718 & $P 4 / m m m ~ \# 123$ & $35 \%$ & $\begin{array}{l}\text { composition not found, nearest are } \operatorname{Sr}_{28} \operatorname{In}_{11} \text { (space group } \\
\operatorname{Imm} 2 \text { ) and } \mathrm{Sr} \text { (space group } F m \overline{3} m \text { ) }\end{array}$ \\
\hline $\mathrm{CoNb}_{2}$ & aflow: f5cc5eaf65e692a9 & $I 4 / m c m ~ \# 140$ & $35 \%$ & $\begin{array}{l}\text { composition not found, nearest are } \mathrm{Nb}_{6.7} \mathrm{Co}_{6.3} \text { (space group } \\
R \overline{3} m, \text { POCC structure) and } \mathrm{Nb}_{0.77} \mathrm{Co}_{0.23} \text { (space group } \\
F m \overline{3} m \text {, POCC structure) }\end{array}$ \\
\hline $\mathrm{Ag}_{3} \operatorname{In}_{2}$ & aflow: 6ee057decaf093d0 & $F d d 2 \# 43$ & $34 \%$ & $\begin{array}{l}\text { composition not found, nearest are } \mathrm{Ag}_{9} \mathrm{In}_{4} \text { (space group } \\
P \overline{4} 3 \mathrm{~m}, \Delta H_{\mathrm{f}}=-21 \mathrm{meV} / \text { atom) and } \mathrm{AgIn}_{2} \text { (space group } \\
I 4 / m c m, \Delta H_{\mathrm{f}}=-53 \mathrm{meV} / \text { atom) }\end{array}$ \\
\hline $\mathrm{AgPt}$ & aflow : 360240 dae $753 \mathrm{fec} 6$ & $P \overline{6} m 2 \# 187$ & $34 \%$ & polymorph found (space group $F m \overline{3} m$, POCC structure) \\
\hline $\mathrm{OsY}_{3}$ & flow: bd3 & Pnma \#62 & $34 \%$ & composition found, one-to-one match \\
\hline $\mathrm{Ag}_{2} \mathrm{Zn}$ & aflow: $1 \mathrm{ba} 6 \mathrm{~b} 4 \mathrm{~b} 5 \mathrm{c} 0 \mathrm{ed} 9788$ & $P \overline{6} 2 m \# 189$ & $33 \%$ & $\begin{array}{l}\text { composition not found, nearest are } \mathrm{Ag} \text { (space group } \\
F m \overline{3} m, \Delta H_{\mathrm{f}}=-4 \mathrm{meV} / \text { atom) and } \mathrm{Ag}_{4.5} \mathrm{Zn}_{4.5} \text { (space group } \\
P \overline{3}, \text { POCC structure) }\end{array}$ \\
\hline MnRh & aflow: $87 \mathrm{~d} 6637 \mathrm{~b} 32224 \mathrm{f} 7 \mathrm{~b}$ & $P m \overline{3} m \# 221$ & $32 \%$ & $\begin{array}{l}\text { polymorph found (space group } P 4 / m m m, \Delta H_{\mathrm{f}}=-156 \\
\text { meV/atom) }\end{array}$ \\
\hline $\mathrm{AgNa}_{2}$ & aflow:f08f 2 f $61 \mathrm{de} 18 \mathrm{aa} 61$ & I4/mcm \#140 & $32 \%$ & $\begin{array}{l}\text { composition not found, nearest are } \mathrm{NaAg}_{2} \text { (space group } \\
F d \overline{3} m, \Delta H_{\mathrm{f}}=-208 \mathrm{meV} / \text { atom) and } \mathrm{Na}(\text { space group } R \overline{3} m)\end{array}$ \\
\hline $\mathrm{BeRe}_{2}$ & aflow : $7 \mathrm{ce} 4 \mathrm{fcc} 3660 \mathrm{c} 16 \mathrm{cf}$ & $I 4 / m c m \# 140$ & $31 \%$ & $\begin{array}{l}\text { composition not found, nearest are } \mathrm{Be}_{2} \mathrm{Re} \text { (space group } \\
P 6_{3} / m m c \text { ) and Re (space group } P 6_{3} / m m c \text { ) }\end{array}$ \\
\hline $\mathrm{As}_{2} \mathrm{Tc}^{\dagger}$ & aflow: e94ab366799a008c & $C 2 / m \# 12$ & $30 \%$ & no diagram \\
\hline $\mathrm{Be}_{2} \mathrm{Mn}^{\dagger}$ & aflow:eec & $P 6_{3} / m m c \# 194$ & $30 \%$ & no diagram \\
\hline & aflow: 6 & $P 4 / m m m ~ \# 123$ & $29 \%$ & polymorph found (space group $F m \overline{3} m$, POCC structure) \\
\hline $\mathrm{Nb}_{5} \mathrm{Re}_{24}$ & aflow: ca051dbe25c55b92 & $I \overline{4} 3 m \# 217$ & $29 \%$ & $\begin{array}{l}\text { composition not found, nearest are } \mathrm{Nb}_{0.25} \mathrm{Re}_{0.75} \text { (space } \\
\text { group } I \overline{4} 3 m \text {, POCC structure) and } \mathrm{Nb}_{0.01} \mathrm{Re}_{0.99} \text { (space } \\
\text { group } P 6_{3} / m m c \text {, POCC structure) }\end{array}$ \\
\hline $\mathrm{La}_{3} \mathrm{Os}^{\dagger}$ & aflow:a9daa699 & Pnma \#62 & $28 \%$ & no diagram \\
\hline & aflow: $8 c e 8$ & $F \overline{4} 3 m \# 216$ & $28 \%$ & composition found, one-to-one match \\
\hline $\mathrm{Ir}_{8} \mathrm{Ru}$ & aflow : $487 \mathrm{f} 7 \mathrm{cf} 6 \mathrm{c} 3 \mathrm{fb} 13 \mathrm{f} 0$ & I4/ $\mathrm{mmm} \# 139$ & $27 \%$ & $\begin{array}{l}\text { composition not found, nearest are Ir (space group } F m \overline{3} m \text { ) } \\
\text { and } \mathrm{Ru}_{0.3} \operatorname{Ir}_{0.7} \text { (space group } F m \overline{3} m \text {, POCC structure) }\end{array}$ \\
\hline $\operatorname{InK}$ & aflow: $66 \mathrm{af} 8171 \mathrm{e} 22 \mathrm{dc} 212$ & $C 2 / m \# 12$ & $27 \%$ & $\begin{array}{l}\text { composition not found, nearest are } \mathrm{K}_{8} \operatorname{In}_{11} \text { (space group } \\
R \overline{3} c \text { ) and } \mathrm{K} \text { (space group } \operatorname{Im} \overline{3} m \text { ) }\end{array}$ \\
\hline PtRh & aflow : 6236a78ecf069d13 & $I 4_{1} /$ amd \#141 & $27 \%$ & polymorph found (space group $F m \overline{3} m$, POCC structure) \\
\hline
\end{tabular}

TABLE 1. The 25 binary phases predicted to be most stable by AFLOW-CHULL. Phases with equivalent structures in the AFLOW ICSD catalog are excluded. The list is sorted by the ratio between the stability criterion $\left(\delta_{\mathrm{sc}}\right)$ and the formation enthalpy $\left(H_{\mathrm{f}}\right)$ (shown as a percentage). ${ }^{\dagger}$ indicates no corresponding binary phase diagram is available on the ASM Alloy Phase Diagram database [5]. POCC denotes a partially-occupied structure [40]. Comparisons with the ASM database include phases that are observed at high temperatures and pressures.

treme contradiction in the topology of the hull, and thus, nearby phases are also analyzed. For the Be-Re system, though $\mathrm{BeRe}_{2}$ has not been observed, both $\mathrm{Be}_{2} \mathrm{Re}$ and $\mathrm{Re}$ are successfully identified. Most of the remaining phases show the nearest phase to be a disordered (partially occupied) structure, which are entirely excluded from the AFLOW.org repository. Addressing disorder is a particularly challenging task in ab-initio studies. However, re- cent high-throughput techniques [40] show promise for future investigations and will be integrated in future releases of the code.

Among the most stable ternary phases, only one system appears to have an available phase diagram in the ASM database, Ag-In-Zr. In this case, the composition of $\mathrm{Ag}_{2} \mathrm{InZr}$ is not observed and the nearest stable phases include POCC structures and $\mathrm{AgZr}_{5} \mathrm{In}_{3}$, which has not 


\begin{tabular}{|c|c|c|c|c|}
\hline compound & auid & laxed space group & $\delta_{\mathrm{sc}} / H_{\mathrm{f}}$ & comparison with ASM Alloy Phase Diagrams [5] \\
\hline $\mathrm{MgSe}_{2} \mathrm{Zn}_{2}^{\dagger}$ & aflow:df0cdf0f1ad3110d & $F m m m \# 69$ & $58 \%$ & $\begin{array}{l}\text { no diagram, two of three binary phase diagrams found (no } \\
\text { Mg-Se) }\end{array}$ \\
\hline $\mathrm{Be}_{4} \mathrm{OsTi}^{\dagger}$ & aflow: 38c259a917a8a6d7 & $F \overline{4} 3 m \# 216$ & $38 \%$ & $\begin{array}{l}\text { no diagram, two of three binary phase diagrams found (no } \\
\text { Be-Os) }\end{array}$ \\
\hline $\mathrm{Be}_{4} \mathrm{OsV}^{\dagger}$ & aflow : 4e5711451dc4b601 & $F \overline{4} 3 m \# 216$ & $38 \%$ & $\begin{array}{l}\text { no diagram, two of three binary phase diagrams found (no } \\
\text { Be-Os) }\end{array}$ \\
\hline $\mathrm{Ag}_{2} \operatorname{InZr}$ & aflow : $1684 \mathrm{c} 02 \mathrm{e} 75 \mathrm{~b} 0 \mathrm{~d} 950$ & $F m \overline{3} m \# 225$ & $35 \%$ & $\begin{array}{l}\text { composition not found, nearest are } \mathrm{Ag}_{0.835} \operatorname{In}_{0.165} \text { (space } \\
\text { group } F m \overline{3} m, \text { POCC structure), } \operatorname{AgZr}_{5} \operatorname{In}_{3} \text { (space group } \\
P 6_{3} / m c m \text { ), and } Z_{0.5} \operatorname{In}_{0.5} \text { (space group } F m \overline{3} m, \text { POCC } \\
\text { structure) }\end{array}$ \\
\hline $\mathrm{Be}_{4} \mathrm{RuTi}^{\dagger \ddagger}$ & aflow:b85addbb42c47ae9 & $F \overline{4} 3 m \# 216$ & $32 \%$ & m, all three binary phase diagrams found \\
\hline $\mathrm{Ti}^{\dagger \ddagger}$ & aflow: cabc & $F \overline{4} 3 m \# 216$ & $29 \%$ & inary pha \\
\hline $\mathrm{Be}_{4} \mathrm{ReV}^{\dagger \ddagger}$ & aflow : 7010472778d429f7 & $F \overline{4} 3 m \# 216$ & $29 \%$ & binary pha \\
\hline $\mathrm{Ba}_{2} \mathrm{RhZn}^{\dagger}$ & aflow: e4cc9eea02d9d303 & $C m \# 8$ & $29 \%$ & $\begin{array}{l}\text { o diagram, two of three binary phase diagrams found (no } \\
\text { a-Rh) }\end{array}$ \\
\hline $\mathrm{Be}_{4} \mathrm{HfOs}^{\dagger}$ & aflow : 2 ace 5 c5383f 8 ea 10 & $F \overline{4} 3 m \# 216$ & $27 \%$ & gram, two of three binary phase diagrams found (no \\
\hline $\mathrm{Be}_{4} \operatorname{ReTi}^{\dagger \ddagger}$ & aflow: de79192a0c4e751f & $F \overline{4} 3 m \# 216$ & $27 \%$ & ram, all three binary phase diagrams found \\
\hline $\mathrm{Be}_{4} \mathrm{TcV}^{\dagger}$ & aflow: d484b95ba623f $9 f 7$ & $F \overline{4} 3 m \# 216$ & $27 \%$ & gram, two of three binary phase diagrams found (no \\
\hline $\mathrm{Be}_{4} \mathrm{TcTi}^{\dagger}$ & aflow: c13660b990eb9570 & $F \overline{4} 3 m \# 216$ & $27 \%$ & gram, two of three binary phase diagrams found (no \\
\hline $\mathrm{Be}_{4} \mathrm{RuV}^{\dagger \ddagger}$ & aflow: $07840 \mathrm{~d} 9 \mathrm{e} 13694 \mathrm{f} 7 \mathrm{e}$ & $F \overline{4} 3 m \# 216$ & $27 \%$ & \\
\hline & aflow: 57 & $F \overline{4} 3 m \# 216$ & $26 \%$ & \\
\hline $\mathrm{Be}_{4} \mathrm{MnTi}^{\dagger}$ & aflow: 9a10dd8a8224e158 & $F \overline{4} 3 m \# 216$ & $26 \%$ & ) \\
\hline $\mathrm{Be}_{4} \mathrm{OsZr}^{\dagger}$ & aflow: de 412213 bdef bd14 & $F \overline{4} 3 m \# 216$ & $26 \%$ & gram, two of three binary phase diagrams found (no \\
\hline $\mathrm{Be}_{4} \operatorname{IrTi}^{\dagger}$ & aflow:07bcc161f57da109 & $F \overline{4} 3 m \# 216$ & $26 \%$ & $\begin{array}{l}\text { no diagram, two of three binary phase diagrams found (no } \\
\text { Be-Ir) }\end{array}$ \\
\hline $\mathrm{Mg}_{2} \mathrm{ScTl}^{\dagger}$ & aflow: 90 b98cdcd6eea146 & $P 4 / m m m \# 123$ & $25 \%$ & $\begin{array}{l}\text { no diagram, two of three binary phase diagrams found (no } \\
\mathrm{Sc}-\mathrm{Tl} \text { ) }\end{array}$ \\
\hline $\mathrm{Be}_{4} \mathrm{MnV}^{\dagger}$ & aflow: 086b4a89f8d62804 & $F \overline{4} 3 m \# 216$ & $25 \%$ & $\begin{array}{l}\text { no diagram, two of three binary phase diagrams found (no } \\
\text { Be-Mn) }\end{array}$ \\
\hline $\mathrm{AuBe}_{4} \mathrm{Cu}^{\dagger \ddagger}$ & flow: & $F \overline{4} 3 m \# 216$ & $25 \%$ & \\
\hline & aflow: d7fed8d4996290f4 & $F \overline{4} 3 m \# 216$ & $24 \%$ & three binary pha \\
\hline $\mathrm{Be}_{4} \mathrm{RhTi}^{\dagger}$ & aflow: faa814b1222e8aea & $F \overline{4} 3 m \# 216$ & $21 \%$ & $\begin{array}{l}\text { no diagram, two of three binary phase diagrams found (no } \\
\text { Be-Rh) }\end{array}$ \\
\hline${ }_{14} \mathrm{Hf}^{\dagger \ddagger}$ & flow: 2 & $F \overline{4} 3 m$ & $21 \%$ & no diagram, all three binary phas \\
\hline $\mathrm{Mg}_{2} \mathrm{SeZn}_{2}^{\dagger}$ & aflow: ab57b1ae74f4c6d4 & Fmmm \#69 & $21 \%$ & $\begin{array}{l}\text { no diagram, two of three binary phase diagrams found (no } \\
\text { Mg-Se) }\end{array}$ \\
\hline $\mathrm{AuCu}_{4} \mathrm{Zr}^{\dagger \ddagger}$ & aflow: $6661 \mathrm{fa} 448 \mathrm{e} 5903 \mathrm{a} 5$ & $F \overline{4} 3 m \# 216$ & $20 \%$ & no diagram, all three binary phase diagrams found \\
\hline
\end{tabular}

TABLE 2. The 25 ternary phases predicted to be most stable by AFLOW-CHULL. Phases with equivalent structures in the AFLOW ICSD catalog are excluded. The list is sorted by the ratio between the stability criterion $\left(\delta_{\mathrm{sc}}\right)$ and the formation enthalpy $\left(H_{\mathrm{f}}\right)$ (shown as a percentage). ${ }^{\dagger}$ indicates no corresponding ternary phase diagram is available on the ASM Alloy Phase Diagram database [5], while ${ }^{\ddagger}$ indicates all three relevant binaries are available. Comparisons with the ASM database include phases that are observed at high temperatures and pressures.

yet been included the AFLOW.org repository. All other ternary systems are entirely unexplored, suggesting an excellent opportunity for informatics-based phase diagrams to pave the path toward discovery. In particular, ternary phases with all three binary phase diagrams available are denoted with the symbol ${ }^{\ddagger}$, suggesting experimental feasibility.

A striking feature of Table 2 is that most of the stable structures are found to be in space group $F \overline{4} 3 m \# 216$. This structure has a face-centered cubic lattice with sym- metry operations that include a four-fold rotation about the $<001>$ axes, a three-fold rotation about the $<111>$ axes, and no inversion. Further study reveals that these phases, as well as $F m \overline{3} m \# 225 \mathrm{Ag}_{2} \mathrm{InZr}$, can be obtained from the "quaternary-Heusler" structure, LiMgPdSn $[72,73]$ (Figure 7(a)). The prototype can be considered a $2 \times 2 \times 2$ supercell of the body-centered cubic structure. The $\mathrm{Sn}, \mathrm{Mg}, \mathrm{Au}$ and $\mathrm{Li}$ atoms all sit a different Wyckoff positions of space group $F \overline{4} 3 \mathrm{~m}$ and each atom has two sets of nearest neighbors, each four-fold coor- 
a

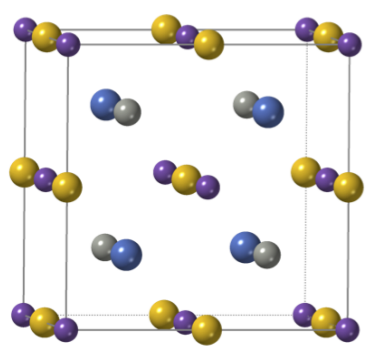

b

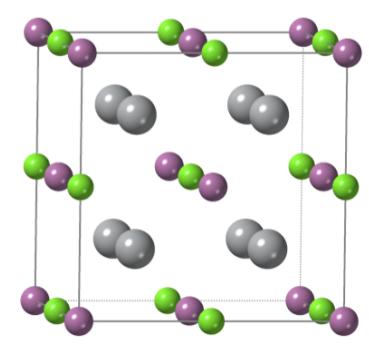

c

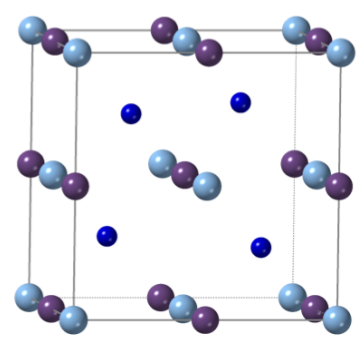

d

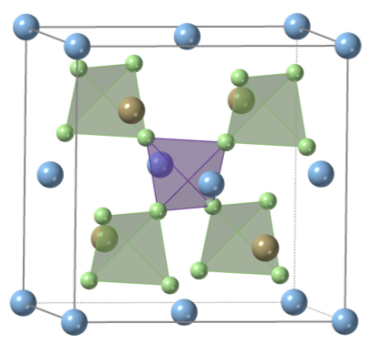

FIG. 7. Illustration of the most prevalent stable ternary structures. (a) The conventional cubic cell of the "quaternary-

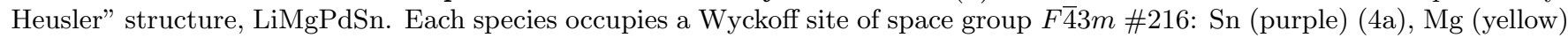
(4b), Pd (gray) (4c), and Li (blue) (4d). (b) The conventional cubic cell of the Heusler structure, here represented by $\mathrm{Ag}_{2} \mathrm{InZr}_{\mathrm{Z}}$ Each species occupies a Wyckoff site of space group $F m \overline{3} m$ \#225: In (pink) (4a), Zr (green) (4b), Ag (light gray) (8c). (c) The conventional cubic cell of the half-Heusler $C 1_{b}$ structure, here represented by AsCoTi. Each species occupies a Wyckoff site of space group $F \overline{4} 3 m$ \#216: Ti (light blue) (4a), As (purple) (4b), Co (dark blue) (4c). The (4d) site is empty. (d) The conventional cubic cell of the $C 15_{b}$-type crystal, here represented by $\mathrm{Be}_{4} \mathrm{OsTi}$. Each species occupies a Wyckoff site of space group $F \overline{4} 3 m$ \#216: Ti (light blue) (4a), Os (brown) (4c), and Be (light green) (8e). The (4d) site is empty, and the Be atoms form a tetrahedron centered around the (4b) site of $(\mathbf{a})$.

dinated. Various decorations of these Wyckoff positions generate the other structures:

- By decorating two second-neighbor atoms identically, a Heusler alloy forms (Strukturbericht symbol $L 2_{1}$ ) [43, 74]. For example, the following substitutions generate $\mathrm{Ag}_{2} \mathrm{InZr}($ Figure $7(\mathrm{~b})): \mathrm{Pd} \rightarrow \mathrm{Ag}, \mathrm{Li} \rightarrow \mathrm{Ag}, \mathrm{Sn} \rightarrow \mathrm{In}$, and $\mathrm{Mg} \rightarrow \mathrm{Zr}$. Since the crystal now has an inversion center, the space group becomes $F m \overline{3} m \# 225$. As in $\mathrm{LiMgPdSn}$, each atom has two sets of four-fold coordinated nearest neighbors, each arranged as a tetrahedron. Now, however, one species (Ag) has secondneighbors of the same type.

- By removing the Li atom completely, a half-Heusler forms $\left(C 1_{b}\right)[43,75]$. There are two half-Heusler systems in Table 2: AsCoTi (Figure 7(c)) and BiRhZr. The structure does differ from that of LiMgPdSn and $L 2_{1}$, as the $\mathrm{Ag}$ and $\mathrm{Ti}$ atoms are four-fold coordinated, with only Co having the coordination seen in the previous structures.

- The majority of structures in Table 2 are type $C 15_{b}$, prototype $\mathrm{AuBe}_{5}[43,76]$ (AFLOW prototype: AB5_cF24_216_a_ce [77]), shown in Figure 7(d). Compared to the $C 1_{b}, C 15_{b}$ contains an (8e) Wyckoff position forming a tetrahedra centered around the (4b) Wyckoff position. Replacing the tetrahedra with a single atom returns the $C 1_{b}$ structure.

Hence, of the 25 most stable ternary structures, 21 are of related structure.

Sampling bias likely plays a role in the high prominence of space group $F \overline{4} 3 m$ \#216 structures in Table 2, but cannot fully account for the anomaly. Space group $F \overline{4} 3 m$ \#216 constitutes about $17 \%$ of the LIB3 catalog, containing the bulk of the AFLOW.org repository (at over 1.4 million ternary systems) generated largely by small structure prototypes. For context, space group $F \overline{4} 3 m$ \#216 is ranked about twentieth of the most common space groups in the ICSD [78], appearing in about
$1 \%$ of all entries. Further exploration of larger structure ternary prototypes covering the full range of space groups is needed to fully elucidate the nature of this structure's stability.

The regular-, inverse-, and half-Heusler prototypes were added to LIB3 recently for the exploration of new magnets, of which two were discovered [4]. Indeed, these structures have been a particularly fruitful addition to the AFLOW.org repository, though are still much unexplored. The fully sorted lists of stable binary and ternary phases are presented in the Supporting Information.

\section{CONCLUSIONS}

Thermodynamics is a critical step for any effective materials design workflow. Being a collective characterization, thermodynamics requires comparisons between many configurations of the system. The availability of large databases [8-15] allows the construction of computationally-based phase diagrams. AFLOWCHULL presents a complete software infrastructure including flexible protocols for data retrieval, analysis, and validation $[12,44]$. The module is exhaustively applied to the AFLOW.org repository and rapidly identifies several new candidate phases: 18 promising $C 15_{b}$-type structures and two half-Heuslers. The extension of AFLOWCHULL to repositories beyond AFLOW.org is trivial and can easily be performed following the open-source C++ code and/or python module. Computational platforms such as AFLOW-CHULL are valuable tools for guiding synthesis, including high-throughput and even autonomous approaches [79-82]. 


\section{SUPPORTING INFORMATION}

The material includes i. a snapshot (inventory) of binary and ternary alloy systems available in the AFLOW.org repository, ii. a full list of stable phases ranked by their relative stability criterion, iii. example scripts illustrating how to employ AFLOW-CHULL from within a Python environment, iv. a thorough thermodynamic characterization of 493 binary systems, and v. 861 ternary systems.

\section{Appendix: AFLOW-CHULL manual}

Command-line options. AFLOW-CHULL is an integrated module of the AFLOW ab-initio framework which runs on any UNIX-like computer, including those running macOS. The most up-to-date binary can be downloaded from materials.duke.edu/AFLOW/: current version 3.1.200. AFLOW-CHULL only depends on the compiled binary executable and an internet connection, as all data is retrieved and analyzed in-situ. The default output option also requires the IATEX package. The output in this work is compiled using pdf $\mathrm{T}_{\mathrm{E}} \mathrm{X}$, Version 3.14159265-2.6-1.40.18 (TEX Live 2017).

The commands are as follows:

Primary commands:

- aflow --chull --alloy=InNiY

- Calculates and returns the convex hull for system In-Ni-Y.

- aflow --chull --alloy=InNiY --stability_criterion=aflow:60a36639191c0af8

- Calculates and returns the stability criterion for $\mathrm{InNi}_{4} \mathrm{Y}$. The structure and relevant duplicates (if any) are removed simultaneously.

- aflow --chull --alloy=InNiY --hull_formation_enthalpy=0.25,0.25

- Calculates and returns the formation enthalpy of the minimum energy surface at $\operatorname{In}_{0.25} \mathrm{Ni}_{0.25} \mathrm{Y}_{0.5}$. The input composition is specified by implicit coordinates (refer to Equation 4), where the last coordinate offers an optional energetic shift.

- aflow --chull --usage

- Prints full set of commands to the screen.

- aflow --readme=chull

- Prints a verbose manual (commands and descriptions) to the screen.

General options:

- - -output=pdf

- Selects the output format. Options include: pdf, json, txt, and all. For multiple output, provide a commaseparated value list. A file with the corresponding extension is created, e.g., aflow_InNiY_hull.pdf.

- --destination=\$HOME/

- Sets the output path to \$HOME. All output will be redirected to this destination.

- - keep=log

- Creates a log file with verbose output of the calculation, e.g., aflow_InNiY_hull.log.

Loading options:

- --load_library=icsd

- Limits the catalogs from which entries are loaded. Options include: icsd, lib1, lib2, and lib3. For multiple catalogs, provide a comma-separated value list.

- --load_entries_entry_output

- Prints verbose output of the entries loaded. This output is included in the log file by default.

- --neglect=aflow : 60a36639191c0af8, aflow : 3f24d2be765237f1, . .

- Excludes individual points from the convex hull calculation.

- --see_neglect

- Prints verbose output of the entries neglected from the calculation, including ill-calculated entries, duplicates, outliers, and those requested via --neglect.

- - -remove_extreme_points=-1000

- Excludes all points with formation enthalpies below $-1000 \mathrm{meV} /$ atom.

- --include_paw_gga

- Includes all entries calculated with PAW-GGA (in addition to those calculated with PAW-PBE). PAW-GGA

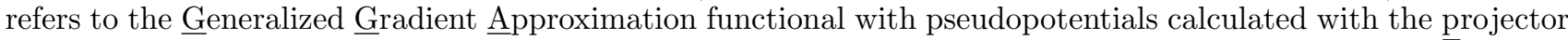
augmented wave method. This flag is needed to generate Figure 4(f).

Analysis options:

- --skip_structure_comparison

- Avoids robust determination of structures equivalent to stable phases (speed). 
- --skip_stability_criterion_analysis

- Avoids determination of the stability criterion of stable phases (speed).

- --include_unreliable_hulls

- Proceeds to calculate the hull in the event that it is determined unreliable (fewer than 200 entries).

- --include_outliers

- Avoids the exclusion of outliers.

- - force

- Forces an output, ignoring all warnings. This flag is needed to generate Figure 4(f).

PDF/LATEX options:

- --image_only

- Creates a PDF with the hull illustration only.

- --document_only

- Creates a PDF with the thermodynamic report only. Default for dimensions $N>3$.

- - -keep=tex

- Saves the $\mathrm{LT}_{\mathrm{E} X} \mathrm{X}$ input file (deleted by default), allowing for customization of the resulting PDF, e.g., aflow_InNiY_hull.tex.

- --latex_interactive

- Displays the LATEX compilation output and enables interaction with the program.

- --plot_iso_max_latent_heat

- Plots the entropic temperature envelopes shown in Figure 4(f). Limited to binary systems only.

AFLOWrc options. Herein we introduce the .aflow.rc file, a new protocol for specifying AFLOW default options. The file emulates the bashrc script that is run in Bash (Bourne again shell) in an interactive environment. The .aflow.rc file is automatically created in $\$ H O M E$ if one is not already present.

Relevant AFLOW-CHULL options include:

- DEFAULT_CHULL_ALLOWED_DFT_TYPES="PAW_PBE"

- Description: Defines the allowed entries based on density functional theory (DFT) calculation type (commaseparated value). Options include: US, GGA, PAW_LDA, PAW_GGA, PAW_PBE, GW, and HSE06 [9].

- Type: string

- DEFAULT_CHULL_ALLOW_ALL_FORMATION_ENERGIES=0

- Description: Allows all entries independent of DFT calculation type [9].

- Type: 0 (false) or 1 (true)

- DEFAULT_CHULL_COUNT_THRESHOLD_BINARIES $=200$

- Description: Defines the minimum number of entries for a reliable binary hull.

- Type: integer

- DEFAULT_CHULL_PERFORM_OUTLIER_ANALYSIS=1

- Description: Enables determination of outliers.

- Type: 0 (false) or 1 (true)

- DEFAULT_CHULL_OUTLIER_ANALYSIS_COUNT_THRESHOLD_BINARIES=50

- Description: Defines the minimum number of entries for a reliable outlier analysis. Only phases stable with respect to their end-members are considered for the outlier analysis (below the zero $H_{\mathrm{f}}$ tie-line).

- Type: integer

- DEFAULT_CHULL_OUTLIER_MULTIPLIER=3.25

- Description: Defines the bounds beyond the interquartile range for which points are considered outliers [62].

- Type: double

- DEFAULT_CHULL_LATEX_PLOT_UNARIES=0

- Description: Incorporates the end-members in the convex hull illustration.

- Type: 0 (false) or 1 (true)

- DEFAULT_CHULL_LATEX_PLOT_OFF_HULL=-1

- Description: Incorporates unstable phases in the convex hull illustration, but excludes phases unstable with respect to their end-members (above the zero $H_{\mathrm{f}}$ tie-line). Only three values are accepted: -1 (default: true for 2-dimensional systems, false for 3-dimensional systems), 0 (false), 1 (true).

- Type: 1 (default), 0 (false), or 1 (true)

- DEFAULT_CHULL_LATEX_PLOT_UNSTABLE=0

- Description: Incorporates all unstable phases in the convex hull illustration.

- Type: 0 (false) or 1 (true)

- DEFAULT_CHULL_LATEX_FILTER_SCHEME=" " 
- Description: Defines exclusion scheme for the convex hull illustration. In contrast to --neglect, this scheme is limited only to the illustration, points are still included in the analysis/report. The following strings are accepted: Z-axis (also Energy-axis) or Distance. Z-axis refers to a scheme that eliminates structures from the illustration based on their formation enthalpy. On the other hand, Distance refers to a scheme that eliminates structures from the illustration based on their distances from the hull. The criteria (value) for elimination is defined by DEFAULT_CHULL_LATEX_FILTER_VALUE.

- Type: string

- DEFAULT_CHULL_LATEX_FILTER_VALUE=50

- Description: Defines the value beyond which points are excluded per the scheme defined with DEFAULT_CHULL_LATEX_FILTER_SCHEME. In this case, AFLOW-CHULL would filter points with energies greater than $50 \mathrm{meV}$.

- Type: double

- DEFAULT_CHULL_LATEX_COLOR_BAR=1

- Description: Defines whether to show the color bar graphic. Colors can still be incorporated without the color bar graphic.

- Type: 0 (false) or 1 (true)

- DEFAULT_CHULL_LATEX_HEAT_MAP=1

- Description: Defines whether to color the facets with heat maps illustrating their depth (3-dimensional illustration only).

- Type: 0 (false) or 1 (true)

- DEFAULT_CHULL_LATEX_COLOR_GRADIENT=1

- Description: Defines whether to incorporate a color scheme at all in the illustration. Turning this flag off will also turn off DEFAULT_CHULL_LATEX_COLOR_BAR and DEFAULT_CHULL_LATEX_HEAT_MAP.

- Type: 0 (false) or 1 (true)

- DEFAULT_CHULL_LATEX_COLOR_MAP=" "

- Description: Defines the color map, options are presented in Ref. [83]. Default is $\mathrm{rgb}(0 \mathrm{pt})=(0.035,0.270,0.809) ; \mathrm{rgb}(63 \mathrm{pt})=(1,0.644,0)$.

- Type: string

- DEFAULT_CHULL_LATEX_LINKS=1

- Description: Defines the links scheme. True/false, i.e., 0/1, will toggle all links on/off. 2 enables external hyperlinks only (no links to other sections of the PDF). 3 enables internal links only (no links to external pages).

- Type: 0 (false), 1 (true), 2 (external-only), or 3 (internal-only)

- DEFAULT_CHULL_LATEX_LABEL_NAME=" "

- Description: Defines the labeling scheme for phases shown on the convex hull. By default, the compound labels are shown, while the prototype label can also be specified. Also acceptable: both and none.

- Type: string

- DEFAULT_CHULL_LATEX_META_LABELS=0

- Description: Enables verbose labels, including compound, prototype, $H_{\mathrm{f}}, T_{\mathrm{S}}$, and $\Delta H_{\mathrm{f}}$. Warning, significant overlap of labels should be expected.

- Type: 0 (false) or 1 (true)

- DEFAULT_CHULL_LATEX_LABELS_OFF_HULL=0

- Description: Enables labels for unstable points.

- Type: 0 (false) or 1 (true)

- DEFAULT_CHULL_LATEX_HELVETICA_FONT $=1$

- Description: Switches the font scheme from Computer Modern (default) to Helvetica.

- Type: 0 (false) or 1 (true)

- DEFAULT_CHULL_LATEX_FONT_SIZE=" "

- Description: Defines the font size of the labels on the convex hull illustration. Warning, other settings may override this default. Options include: tiny, scriptsize, footnotesize, small, normalsize, large (default), Large, LARGE, huge, and Huge.

- Type: string

- DEFAULT_CHULL_LATEX_ROTATE_LABELS=1

- Description: Toggles whether labels are appropriately rotated.

- Type: 0 (false) or 1 (true)

- DEFAULT_CHULL_LATEX_BOLD_LABELS=-1

- Description: Toggles whether labels are bolded. Only three values are accepted: -1 (default: false unless phase is a ternary), 0 (false), 1 (true).

- Type: -1 (default), 0 (false), or 1 (true) 
Python environment. A module has been created that employs AFLOW-CHULL within a Python environment. The module and its description closely follow that of the AFLOW-SYM Python module [45]. It connects to a local AFLOW installation and imports the AFLOW-CHULL results into a CHull class. A CHull object is initialized with:

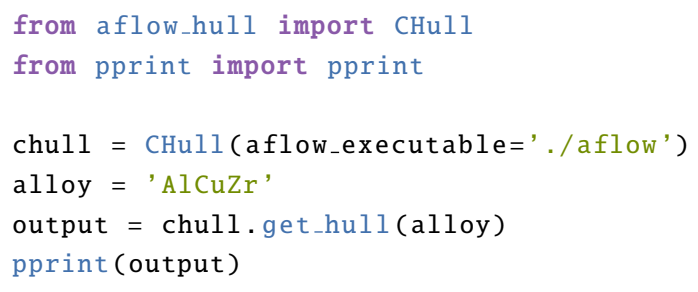

By default, the CHull object searches for an AFLOW executable in the \$PATH. However, the location of an AFLOW executable can be specified as follows:

CHull (aflow_executable=\$HOME/bin/aflow).

The CHull object contains built-in methods corresponding to the command line calls mentioned previously:

- get_hull ('InNiY')

- get_stability_criterion('InNiY', 'aflow:60a36639191c0af8')

- get_hull_energy ('InNiY', [0.25,0.25])

Each method requires an input alloy string. get_stability_criterion additionally requires a string input for the auid, while get_hull_energy takes an array of doubles as its input for the composition.

Python module. The module to run the aforementioned AFLOW-CHULL commands is provided below. This module can easily be modified to incorporate additional options.

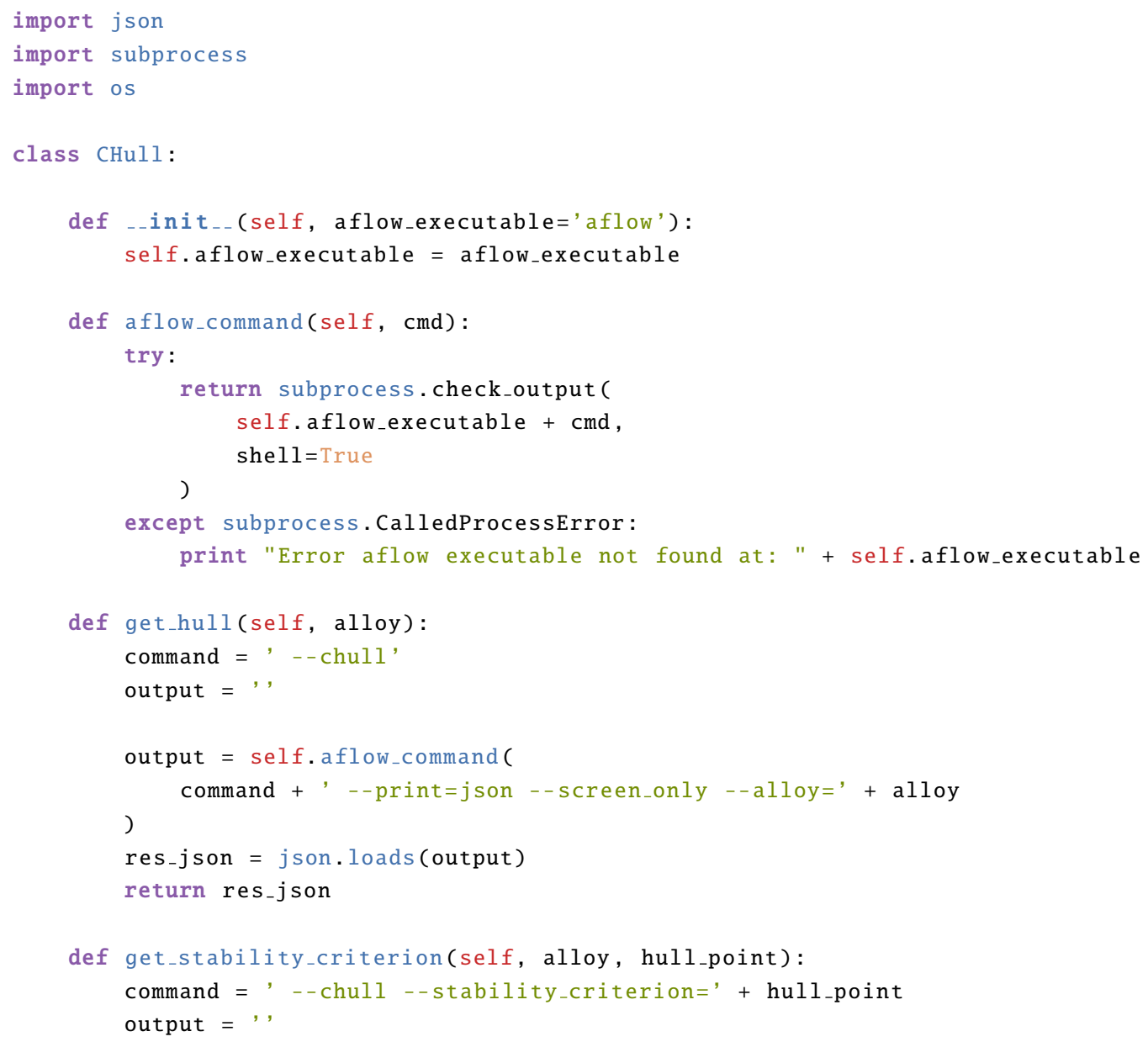




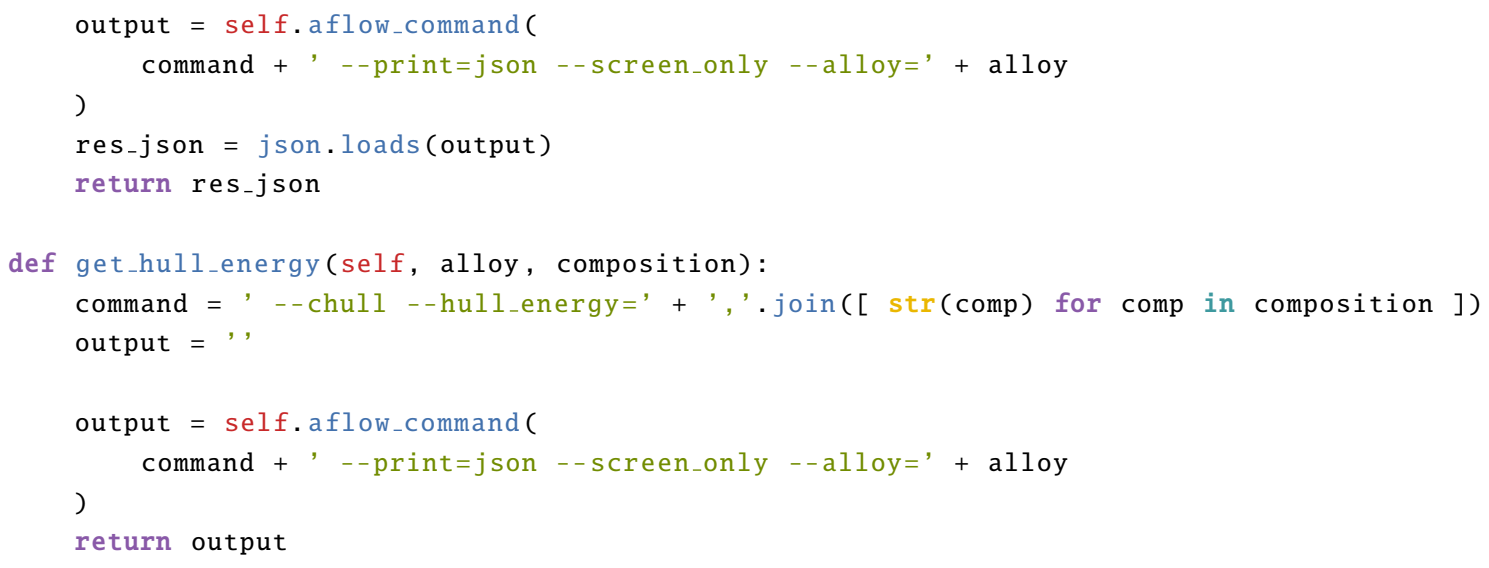

Output list. This section details the output fields for the thermodynamic analysis. The lists describe the keywords as they appear in the JSON format. Similar keywords are used for the standard text output.

Points data (points_data).

- auid

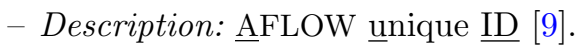

- Type: string

- aurl

- Description: ㅅFLOW uniform resource locator [9].

- Type: string

- compound

- Description: Compound name [9].

- Type: string

- enthalpy_formation_atom

- Description: Formation enthalpy per atom $\left(H_{\mathrm{f}}\right)[9]$.

- Type: double

- Units: $\mathrm{meV} /$ atom

- enthalpy_formation_atom_difference

- Description: Energy driving the decomposition reaction $\left(\Delta H_{\mathrm{f}}\right)$, i.e., the distance to the hull.

- Type: double

- Units: $\mathrm{meV} /$ atom

- entropic_temperature

- Description: The ratio of the formation enthalpy and the ideal mixing entropy $\left(T_{\mathrm{S}}\right)$ [29]. This term defines the ideal "iso-max-latent-heat" lines of the grand-canonical ensemble [29, 53]. Refer to Figure 4.

- Type: double

- Units: Kelvin

- equivalent_structures_auid

- Description: auid of structurally equivalent entries. This analysis is limited to stable phases only.

- Type: array of strings

- ground_state

- Description: True for stable phases, and false otherwise.

- Type: boolean

- icsd_canonical_auid

- Description: auid of an equivalent ICSD entry. If there are multiple equivalent ICSD entries, the one with the lowest number designation is chosen (original usually). This analysis is limited to stable phases only.

- Type: string

- icsd_ground_state

- Description: True for stable phases with an equivalent ICSD entry, and false otherwise.

- Type: boolean

- phases_decomposition_auid

- Description: auid of the products of the decomposition reaction (stable phases). This analysis is limited to 
unstable phases only.

- Type: array of strings

- phases_decomposition_coefficient

- Description: Coefficients of the decomposition reaction normalized to reactant, i.e., $\mathbf{N}$ from Equation 9. Hence, the first entry is always 1 . This analysis is limited to unstable phases only.

- Type: array of doubles

- phases_decomposition_compound

- Description: compound of the products of the decomposition reaction (stable phases). This analysis is limited to unstable phases only.

- Type: array of strings

- phases_equilibrium_auid

- Description: auid of phases in coexistence. This analysis is limited stable phases only.

- Type: array of strings

- phases_equilibrium_compound

- Description: compound of phases in coexistence. This analysis is limited stable phases only.

- Type: array of strings

- prototype

- Description: AFLOW prototype designation [9].

- Type: string

- space_group_orig

- Description: The space group (symbol and number) of the structure pre-relaxation as determined by AFLOWSYM [45].

- Type: string

- space_group_relax

- Description: The space group (symbol and number) of the structure post-relaxation as determined by AFLOWSYM [45].

- Type: string

- spin_atom

- Description: The magnetization per atom for spin polarized calculations [9].

- Type: double

- Units: $\mu_{\mathrm{B}} /$ atom.

- stability_criterion

- Description: A metric for robustness of a stable phase $\left(\delta_{\mathrm{sc}}\right)$, i.e., the distance of a stable phase from the pseudo-hull constructed without it. This analysis is limited to stable phases only.

- Type: double

- Units: $\mathrm{meV} /$ atom

- url_entry_page

- Description: The URL to the entry page: http://aflow.org/material.php?id=aflow:60a36639191c0af8.

- Type: string

Facets data (facets_data).

- artificial

- Description: True if the facet is artificial, i.e., defined solely by artificial end-points, and false otherwise.

- Type: boolean

- centroid

- Description: The centroid of the facet.

- Type: array of doubles

- Units: Stoichiometric-energetic coordinates as defined by Equation 4.

- content

- Description: The content (hyper-volume) of the facet.

- Type: array of doubles

- Units: Stoichiometric-energetic coordinates as defined by Equation 4.

- hypercollinearity

- Description: True if the facet has no content, i.e., exhibits hyper-collinearity, and false otherwise.

- Type: boolean

- Units: Stoichiometric-energetic coordinates as defined by Equation 4.

- normal 
- Description: The normal vector characterizing the facet, i.e., $\mathbf{n}$ in Equation 5.

- Type: double

- Units: Stoichiometric-energetic coordinates as defined by Equation 4.

- offset

- Description: The offset in the hyperplane description of the facet, i.e., D in Equation 5.

- Type: double

- Units: Stoichiometric-energetic coordinates as defined by Equation 4.

- vertical

- Description: True if the facet is vertical along the energetic axis, and false otherwise.

- Type: boolean

- vertices_auid

- Description: auid of the phases that define the vertices of the facet.

- Type: array of strings

- vertices_compound

- Description: compound of the phases that define the vertices of the facet.

- Type: array of strings

- vertices_position

- Description: Coordinates that define the vertices of the facet.

- Type: array of arrays of doubles

- Units: Stoichiometric-energetic coordinates as defined by Equation 4.

Technical support. Functionality requests and bug reports should be posted on the AFLOW Forum aflow.org/forum under the board Thermodynamic analysis.

[1] M. Jansen, A Concept for Synthesis Planning in SolidState Chemistry, Angew. Chem. Int. Ed. 41, 3746-3766 (2002).

[2] R. Potyrailo, K. Rajan, K. Stoewe, I. Takeuchi, B. Chisholm, and H. Lam, Combinatorial and highthroughput screening of materials libraries: Review of state of the art, ACS Comb. Sci. 13, 579-633 (2011).

[3] M. D. Kuz'min, K. P. Skokov, H. Jian, I. Radulov, and O. Gutfleisch, Towards high-performance permanent magnets without rare earths, J. Phys.: Condens. Matter 26, 064205 (2014).

[4] S. Sanvito, C. Oses, J. Xue, A. Tiwari, M. Zic, T. Archer, P. Tozman, M. Venkatesan, J. M. D. Coey, and S. Curtarolo, Accelerated discovery of new magnets in the Heusler alloy family, Sci. Adv. 3, e1602241 (2017).

[5] P. Villars, H. Okamoto, and K. Cenzual, ASM Alloy Phase Diagram Database, http://www1.asminternational.org/AsmEnterprise/APD (2006).

[6] A. Walsh, Inorganic materials: The quest for new functionality, Nat. Chem. 7, 274-275 (2015).

[7] O. Isayev, C. Oses, C. Toher, E. Gossett, S. Curtarolo, and A. Tropsha, Universal fragment descriptors for predicting electronic properties of inorganic crystals, Nat. Commun. 8, 15679 (2017).

[8] S. Curtarolo, W. Setyawan, S. Wang, J. Xue, K. Yang, R. H. Taylor, L. J. Nelson, G. L. W. Hart, S. Sanvito, M. Buongiorno Nardelli, N. Mingo, and O. Levy, AFLOWLIB.ORG: A distributed materials properties repository from high-throughput ab initio calculations, Comput. Mater. Sci. 58, 227-235 (2012).
[9] R. H. Taylor, F. Rose, C. Toher, O. Levy, K. Yang, M. Buongiorno Nardelli, and S. Curtarolo, A RESTful API for exchanging materials data in the AFLOWLIB.org consortium, Comput. Mater. Sci. 93, 178-192 (2014).

[10] C. E. Calderon, J. J. Plata, C. Toher, C. Oses, O. Levy, M. Fornari, A. Natan, M. J. Mehl, G. L. W. Hart, M. Buongiorno Nardelli, and S. Curtarolo, The AFLOW standard for high-throughput materials science calculations, Comput. Mater. Sci. 108 Part A, 233-238 (2015).

[11] F. Rose, C. Toher, E. Gossett, C. Oses, M. Buongiorno Nardelli, M. Fornari, and S. Curtarolo, AFLUX: The LUX materials search API for the AFLOW data repositories, Comput. Mater. Sci. 137, 362-370 (2017).

[12] M. Scheffler, C. Draxl, and Computer Center of the Max-Planck Society, Garching, The NoMaD Repository, http://nomad-repository.eu (2014).

[13] A. Jain, S. P. Ong, G. Hautier, W. Chen, W. D. Richards, S. Dacek, S. Cholia, D. Gunter, D. Skinner, G. Ceder, and K. A. Persson, Commentary: The Materials Project: A materials genome approach to accelerating materials innovation, APL Mater. 1, 011002 (2013).

[14] J. E. Saal, S. Kirklin, M. Aykol, B. Meredig, and C. Wolverton, Materials Design and Discovery with High-Throughput Density Functional Theory: The Open Quantum Materials Database (OQMD), JOM 65, 15011509 (2013).

[15] D. D. Landis, J. S. Hummelshøj, S. Nestorov, J. Greeley, M. Dułak, T. Bligaard, J. K. Nørskov, and K. W. Jacobsen, The Computational Materials Repository, Comput. Sci. Eng. 14, 51-57 (2012). 
[16] G. Pizzi, A. Cepellotti, R. Sabatini, N. Marzari, and B. Kozinsky, AiiDA: automated interactive infrastructure and database for computational science, Comput. Mater. Sci. 111, 218-230 (2016).

[17] C. Nyshadham, C. Oses, J. E. Hansen, I. Takeuchi, S. Curtarolo, and G. L. W. Hart, A computational high-throughput search for new ternary superalloys, Acta Mater. 122, 438-447 (2017).

[18] J. S. Bechtel and A. Van der Ven, First-principles thermodynamics study of phase stability in inorganic halide perovskite solid solutions, Phys. Rev. Mater. 2, 045401 (2018).

[19] W. Li, R. Jacobs, and D. Morgan, Predicting the thermodynamic stability of perovskite oxides using machine learning models, Comput. Mater. Sci. 150, 454-463 (2018).

[20] P. V. Balachandran, A. A. Emery, J. E. Gubernatis, T. Lookman, C. Wolverton, and A. Zunger, Predictions of new $\mathrm{ABO}_{3}$ perovskite compounds by combining machine learning and density functional theory, Phys. Rev. Mater. 2, 043802 (2018).

[21] O. Levy, G. L. W. Hart, and S. Curtarolo, Uncovering Compounds by Synergy of Cluster Expansion and HighThroughput Methods, J. Am. Chem. Soc. 132, 4830-4833 (2010).

[22] O. Levy, G. L. W. Hart, and S. Curtarolo, Hafnium binary alloys from experiments and first principles, Acta Mater. 58, 2887-2897 (2010).

[23] O. Levy, R. V. Chepulskii, G. L. W. Hart, and S. Curtarolo, The New face of Rhodium Alloys: Revealing Ordered Structures from First Principles, J. Am. Chem. Soc. 132, 833-837 (2010).

[24] O. Levy, G. L. W. Hart, and S. Curtarolo, Structure maps for hcp metals from first-principles calculations, Phys. Rev. B 81, 174106 (2010).

[25] O. Levy, M. Jahnátek, R. V. Chepulskii, G. L. W. Hart, and S. Curtarolo, Ordered Structures in Rhenium Binary Alloys from First-Principles Calculations, J. Am. Chem. Soc. 133, 158-163 (2011).

[26] M. Jahnátek, O. Levy, G. L. W. Hart, L. J. Nelson, R. V. Chepulskii, J. Xue, and S. Curtarolo, Ordered phases in ruthenium binary alloys from high-throughput firstprinciples calculations, Phys. Rev. B 84, 214110 (2011).

[27] O. Levy, J. Xue, S. Wang, G. L. W. Hart, and S. Curtarolo, Stable ordered structures of binary technetium alloys from first principles, Phys. Rev. B 85, 012201 (2012).

[28] J. Bloch, O. Levy, B. Pejova, J. Jacob, S. Curtarolo, and B. Hjörvarsson, Prediction and Hydrogen Acceleration of Ordering in Iron-Vanadium Alloys, Phys. Rev. Lett. 108, 215503 (2012).

[29] G. L. W. Hart, S. Curtarolo, T. B. Massalski, and O. Levy, Comprehensive Search for New Phases and Compounds in Binary Alloy Systems Based on PlatinumGroup Metals, Using a Computational First-Principles Approach, Phys. Rev. X 3, 041035 (2013).

[30] S. Barzilai, C. Toher, S. Curtarolo, and O. Levy, Evaluation of the tantalum-titanium phase diagram from abinitio calculations, Acta Mater. 120, 255-263 (2016).

[31] E. Perim, D. Lee, Y. Liu, C. Toher, P. Gong, Y. Li, W. N. Simmons, O. Levy, J. J. Vlassak, J. Schroers, and S. Curtarolo, Spectral descriptors for bulk metallic glasses based on the thermodynamics of competing crystalline phases, Nat. Commun. 7, 12315 (2016).
[32] S. Barzilai, C. Toher, S. Curtarolo, and O. Levy, The effect of lattice stability determination on the computational phase diagrams of intermetallic alloys, J. Alloys Compd. 728, 314-321 (2017).

[33] S. Barzilai, C. Toher, S. Curtarolo, and O. Levy, Molybdenum-titanium phase diagram evaluated from ab initio calculations, Phys. Rev. Mater. 1, 023604 (2017).

[34] A. Hever, C. Oses, S. Curtarolo, O. Levy, and A. Natan, The Structure and Composition Statistics of $6 \mathrm{~A}$ Binary and Ternary Crystalline Materials, Inorg. Chem. 57, 653-667 (2018).

[35] C. M. Rost, E. Sachet, T. Borman, A. Moballegh, E. C. Dickey, D. Hou, J. L. Jones, S. Curtarolo, and J.-P. Maria, Entropy-stabilized oxides, Nat. Commun. 6, 8485 (2015).

[36] Z. Rak, C. M. Rost, M. Lim, P. Sarker, C. Toher, S. Curtarolo, J.-P. Maria, and D. W. Brenner, Charge compensation and electrostatic transferability in three entropystabilized oxides: Results from density functional theory calculations, J. Appl. Phys. 120, 095105 (2016).

[37] Y. Lederer, C. Toher, K. S. Vecchio, and S. Curtarolo, The search for high entropy alloys: a high-throughput abinitio approach, submitted arXiv:1711.03426 (2018).

[38] GNU General Public License, http://www.gnu.org/ licenses.

[39] S. Curtarolo, W. Setyawan, G. L. W. Hart, M. Jahnátek, R. V. Chepulskii, R. H. Taylor, S. Wang, J. Xue, K. Yang, O. Levy, M. J. Mehl, H. T. Stokes, D. O. Demchenko, and D. Morgan, AFLOW: An automatic framework for highthroughput materials discovery, Comput. Mater. Sci. 58, 218-226 (2012).

[40] K. Yang, C. Oses, and S. Curtarolo, Modeling OffStoichiometry Materials with a High-Throughput AbInitio Approach, Chem. Mater. 28, 6484-6492 (2016).

[41] J. Carrete, N. Mingo, S. Wang, and S. Curtarolo, Nanograined Half-Heusler Semiconductors as Advanced Thermoelectrics: An Ab Initio High-Throughput Statistical Study, Adv. Func. Mater. 24, 7427-7432 (2014).

[42] W. Setyawan and S. Curtarolo, High-throughput electronic band structure calculations: Challenges and tools, Comput. Mater. Sci. 49, 299-312 (2010).

[43] M. J. Mehl, D. Hicks, C. Toher, O. Levy, R. M. Hanson, G. L. W. Hart, and S. Curtarolo, The AFLOW Library of Crystallographic Prototypes: Part 1, Comput. Mater. Sci. 136, S1-S828 (2017).

[44] A. R. Supka, T. E. Lyons, L. S. I. Liyanage, P. D'Amico, R. Al Rahal Al Orabi, S. Mahatara, P. Gopal, C. Toher, D. Ceresoli, A. Calzolari, S. Curtarolo, M. Buongiorno

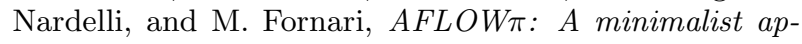
proach to high-throughput ab initio calculations including the generation of tight-binding hamiltonians, Comput. Mater. Sci. 136, 76-84 (2017).

[45] D. Hicks, C. Oses, E. Gossett, G. Gomez, R. H. Taylor, C. Toher, M. J. Mehl, O. Levy, and S. Curtarolo, AFLOW-SYM: platform for the complete, automatic and self-consistent symmetry analysis of crystals, Acta Crystallogr. Sect. A 74, 184-203 (2018).

[46] G. S. Rohrer, M. Affatigato, M. Backhaus, R. K. Bordia, H. M. Chan, S. Curtarolo, A. Demkov, J. N. Eckstein, K. T. Faber, J. E. Garay, Y. Gogotsi, L. Huang, L. E. Jones, S. V. Kalinin, R. J. Lad, C. G. Levi, J. Levy, J.P. Maria, L. Mattos Jr., A. Navrotsky, N. Orlovskaya, C. Pantano, J. F. Stebbins, T. S. Sudarshan, T. Tani, and K. S. Weil, Challenges in Ceramic Science: A Re- 
port from the Workshop on Emerging Research Areas in Ceramic Science, J. Am. Ceram. Soc. 95, 3699-3712 (2012).

[47] C. B. Barber, D. P. Dobkin, and H. Huhdanpaa, The quickhull algorithm for convex hulls, ACM Trans. Math. Soft. 22, 469-483 (1996).

[48] J. Ganguly, Thermodynamics in Earth and Planetary Sciences (Springer-Verlag Berlin Heidelberg, Berlin, 2008), doi:10.1007/978-3-540-77306-1.

[49] L. S. Darken and R. W. Gurry, Physical Chemistry of Metals (McGraw-Hill Book Company, Inc., New York, 1953).

[50] D. A. McQuarrie, Statistical Mechanics (Harper and Row, New York, 1976).

[51] W. S. Massey, Cross Products of Vectors in Higher Dimensional Euclidean Spaces, Am. Math. Mon. 90, 697701 (1983).

[52] D. M. Y. Sommerville, An Introduction to the Geometry of $N$ Dimensions (Dover Publications, Inc., New York, 1929).

[53] J. Yong, Y. Jiang, D. Usanmaz, S. Curtarolo, X. Zhang, L. Li, X. Pan, J. Shin, I. Takeuchi, and R. L. Greene, Robust Topological Surface State of Kondo insulator $\mathrm{SmB}_{6}$ Thin Films, Appl. Phys. Lett. 105, 222403 (2014).

[54] G. Kresse and J. Hafner, Ab initio molecular dynamics for liquid metals, Phys. Rev. B 47, 558-561 (1993).

[55] G. Kresse and J. Hafner, Ab initio molecular-dynamics simulation of the liquid-metal-amorphous-semiconductor transition in germanium, Phys. Rev. B 49, 14251-14269 (1994).

[56] G. Kresse and J. Furthmüller, Efficiency of ab-initio total energy calculations for metals and semiconductors using a plane-wave basis set, Comput. Mater. Sci. 6, 15-50 (1996).

[57] G. Kresse and J. Furthmüller, Efficient iterative schemes for ab initio total-energy calculations using a plane-wave basis set, Phys. Rev. B 54, 11169-11186 (1996).

[58] P. Wisesa, K. A. McGill, and T. Mueller, Efficient generation of generalized Monkhorst-Pack grids through the use of informatics, Phys. Rev. B 93, 155109 (2016).

[59] J. P. Perdew, K. Burke, and M. Ernzerhof, Generalized Gradient Approximation Made Simple, Phys. Rev. Lett. 77, 3865-3868 (1996).

[60] P. E. Blöchl, Projector augmented-wave method, Phys. Rev. B 50, 17953-17979 (1994).

[61] R. H. Taylor, S. Curtarolo, and G. L. W. Hart, Guiding the experimental discovery of magnesium alloys, Phys. Rev. B 84, 084101 (2011).

[62] J. Miller, Short Report: Reaction Time Analysis with Outlier Exclusion: Bias Varies with Sample Size, Q. J. Exp. Psychol. A 43, 907-912 (1991).

[63] C. Leys, C. Ley, O. Klein, P. Bernard, and L. Licata, Detecting outliers: Do not use standard deviation around the mean, use absolute deviation around the median, J. Exp. Soc. Psychol. 49, 764-766 (2013).

[64] D. Hicks, C. Toher, C. De Santo, O. Levy, M. J. Mehl, and S. Curtarolo, AFLOW-XTAL-MATCH: Automated method for quantifying the structural similarity of materials and identifying unique crystal prototypes, in preparation (2018).

[65] H. Burzlaff and Y. Malinovsky, A Procedure for the Classification of Non-Organic Crystal Structures. I. Theoretical Background, Acta Crystallogr. Sect. A 53, 217-224
(1997).

[66] G. Bergerhoff, R. Hundt, R. Sievers, and I. D. Brown, The inorganic crystal structure data base, J. Chem. Inf. Comput. Sci. 23, 66-69 (1983).

[67] A. Belsky, M. Hellenbrandt, V. L. Karen, and P. Luksch, New developments in the Inorganic Crystal Structure Database (ICSD): accessibility in support of materials research and design, Acta Crystallogr. Sect. B 58, 364-369 (2002).

[68] J. Sato, T. Omori, K. Oikawa, I. Ohnuma, R. Kainuma, and K. Ishida, Cobalt-Base High-Temperature Alloys, Science 312, 90-91 (2006).

[69] L. R. Thorne, An Innovative Approach to Balancing Chemical-Reaction Equations: A Simplified MatrixInversion Technique for Determining The Matrix Null Space, arXiv:1110.4321 (2011).

[70] L. N. Trefethen and D. Bau III, Numerical Linear Algebra, Miscellaneous Bks (Society for Industrial and Applied Mathematics, 1997).

[71] S. Kirklin, J. E. Saal, V. I. Hegde, and C. Wolverton, High-throughput computational search for strengthening precipitates in alloys, Acta Mater. 102, 125-135 (2016).

[72] U. Eberz, W. Seelentag, and H.-U. Schuster, Zur Kenntnis farbiger ternärer und quaternärer Zintl-Phasen [Coloured Ternary and Quaternary Zintl-Phases], Z. Naturforsch. B 35, 1341-1343 (1980).

[73] D. Hicks, M. J. Mehl, E. Gossett, C. Toher, O. Levy, R. M. Hanson, G. L. W. Hart, and S. Curtarolo, The AFLOW Library of Crystallographic Prototypes: Part 2, in preparation (2018).

[74] A. J. Bradley and J. W. Rodgers, The Crystal Structure of Heusler Alloys, Proc. R. Soc. A Math. Phys. Eng. Sci. 144, 340-359 (1934).

[75] H. Nowotny and W. Sibert, Ternäre Valenzverbindungen in den Systemen Kupfer(Silber)Arsen(Antimon,Wismut)-Magnesium, Z. Metallkd. 33, 391-394 (1941).

[76] F. W. von Batchelder and R. F. Raeuchle, The tetragonal $\mathrm{MBe}_{12}$ structure of silver, palladium, platinum and gold, Acta Cryst. 11, 122 (1958).

[77] http://aflow.org/CrystalDatabase/AB5_cF24_216_a_ ce.html.

[78] V. S. Urusov and T. N. Nadezhina, Frequency distribution and selection of space groups in inorganic crystal chemistry, J. Struct. Chem. 50, 22-37 (2009).

[79] X. D. Xiang, X. Sun, G. Briceño, Y. Lou, K.-A. Wang, H. Chang, W. G. Wallace-Freedman, S.-W. Chen, and P. G. Schultz, A Combinatorial Approach to Materials Discovery, Science 268, 1738-1740 (1995).

[80] I. Takeuchi, O. O. Famodu, J. C. Read, M. A. Aronova, K. S. Chang, C. Craciunescu, S. E. Lofland, M. Wuttig, F. C. Wellstood, L. Knauss, and A. Orozco, Identification of novel compositions of ferromagnetic shape-memory alloys using composition spreads, Nat. Mater. 2, 180-184 (2003).

[81] H. Koinuma and I. Takeuchi, Combinatorial solid-state chemistry of inorganic materials, Nat. Mater. 3, 429-438 (2004).

[82] S. Curtarolo, G. L. W. Hart, M. Buongiorno Nardelli, N. Mingo, S. Sanvito, and O. Levy, The high-throughput highway to computational materials design, Nat. Mater. 12, 191-201 (2013).

[83] C. Feuersänger, Manual for Package PGFPLOTS, $\operatorname{LTT}_{\mathrm{E} X}(2018)$. 